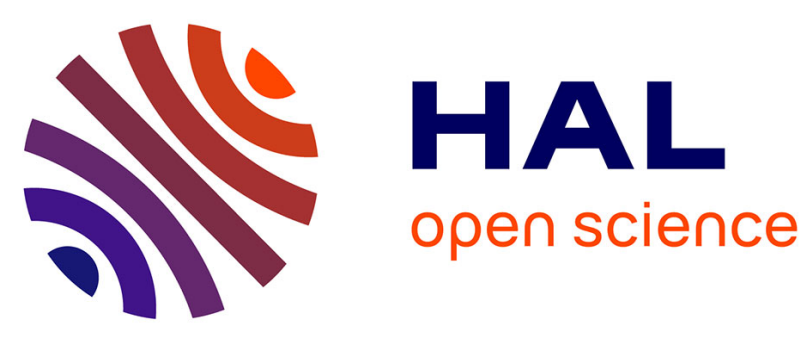

\title{
Physics of vortex merging
}

Patrice Meunier, Stéphane Le Dizès, Thomas Leweke

\section{To cite this version:}

Patrice Meunier, Stéphane Le Dizès, Thomas Leweke. Physics of vortex merging. Comptes Rendus. Physique, 2005, 6, n 4-5, pp.431-450. 10.1016/j.crhy.2005.06.003 . hal-00081677

\section{HAL Id: hal-00081677 https://hal.science/hal-00081677}

Submitted on 27 Jun 2007

HAL is a multi-disciplinary open access archive for the deposit and dissemination of scientific research documents, whether they are published or not. The documents may come from teaching and research institutions in France or abroad, or from public or private research centers.
L'archive ouverte pluridisciplinaire HAL, est destinée au dépôt et à la diffusion de documents scientifiques de niveau recherche, publiés ou non, émanant des établissements d'enseignement et de recherche français ou étrangers, des laboratoires publics ou privés. 


\title{
Physics of vortex merging
}

\author{
P. Meunier, S. Le Dizès, T. Leweke \\ Institut de Recherche sur les Phénomènes Hors Equilibre \\ CNRS / Universités Aix-Marseille I \& II \\ 49, rue Frédéric Joliot Curie, BP 146, F-13384 Marseille Cedex 13, France
}

\begin{abstract}
This article deals with the interaction of co-rotating vortices, in configurations similar to those found in the extended near-wake of typical transport aircraft. The fundamental process of vortex merging is analyzed and modeled in detail in a two-dimensional context, giving insight into the conditions for merging and its physical origin, and yielding predictions for the resulting flow. Three-dimensional effects, in the form an elliptic short-wave instability arising in the initial corotating vortex flow, are described and analyzed theoretically. They are found to cause significant changes in the merging process, like earlier merging and larger final vortex cores. Illustrations from recent experimental, numerical and theoretical studies are given, and the relevance of the results for applications to real aircraft wakes is discussed.
\end{abstract}

Key words: vortex merging, elliptic instability, aircraft wakes

\section{Résumé}

Aspects physiques de la fusion de tourbillons - Cet article traite de l'interaction entre tourbillons co-rotatifs, dans des configurations semblables à celles présentes dans le sillage proche et moyen des avions de transport. Le processus fondamental de fusion des tourbillons est analysé et modélisé en détail dans une description bidimensionnelle, donnant accès à des conditions pour la fusion et son origine physique, ainsi qu'aux propriétés de l'écoulement résultant. Les effets tridimensionnels, sous forme d'une instabilité elliptique à courte longueur d'onde des vortex co-rotatifs initiaux, sont décrits et analysés théoriquement. Ils sont à l'origine de modifications importantes de la fusion, comme un démarrage plus rapide du processus et un vortex final plus gros. Des illustrations d'études expérimentales, numériques et théoriques récentes sont données, et la pertinence des résultats pour des applications aux sillages réalistes des avions est discutée.

Mots-clés: fusion de tourbillons, instabilité elliptique, sillages d'avions

Email address: meunier@irphe.univ-mrs.fr (P. Meunier). 


\section{Introduction}

The merging phenomenon occurs when two vortices of the same sign with (almost) parallel axes, and within a certain critical distance of each other, mix a substantial portion of their core vorticity to become a single vortex. Vortex merging, which is principally a twodimensional process, is one of the fundamental ingredients of fluid motion and plays a major role in a variety of situations, such as decaying two-dimensional turbulence, threedimensional turbulence, and mixing layers, to name a few. Its potential significance covers various fields such as astrophysics, meteorology, and geophysics.[1]

Vortex merging also plays an important role in the context of aircraft trailing wakes. A lifting aircraft wing generates a sheet of longitudinal vorticity, whose structure depends on the lift distribution along the span, dictated by the geometry of the wing and its different elements (flaps, spoilers, engines and nacelles, etc.). In the near field, the vortex sheet quickly rolls up into a set of discrete vortices, which subsequently interact and merge to form a single vortex behind each wing in the aircraft's far wake.

The wake vortex issue is particularly important for the traffic near airports, since aircraft follow each other closely there. Figure 1 shows schematically a typical trailing vortex system generated by an aircraft in take-off or landing configuration. Two strong vortices of comparable strength are generated from the tips of the wing and the lowered flap. These corotating vortices spin around each other by mutual induction, and merge into a single one over a distance of 5-10 wing spans. Issues related to the merging of trailing vortices include the following questions: when and under which conditions do two given vortices merge, in other words: what is the time (distance) to merging? What is the role of three-dimensional effects? What are the characteristics of the merged final vortex? These questions are all relevant for the dynamics and final decay of the counter-rotating vortex pair in the far wake of an aircraft, involving turbulent dissipation and different three-dimensional instabilities, as discussed by Jacquin et al. [2].

The present paper deals with aspects of the dynamics and merging of (nearly) parallel corotating vortices, such as those observed in the extended near wake of a landing aircraft. In section 2, the two-dimensional dynamics are reviewed in detail. Experimental and numerical results will be used to analyze the different stages of merging, establish a merging criterion, including a discussion of the physical origin of merging, and assess the properties of the final vortex. Section 3 is dedicated to three-dimensional effects, in particular a short-wavelength elliptic instability, which can arise in the co-rotating vortices before merging, and which can significantly modify the merging process and the properties of the final vortex. In section 4 , these results are discussed in the context of realistic aircraft wakes, and a conclusion is given in section 5 . 


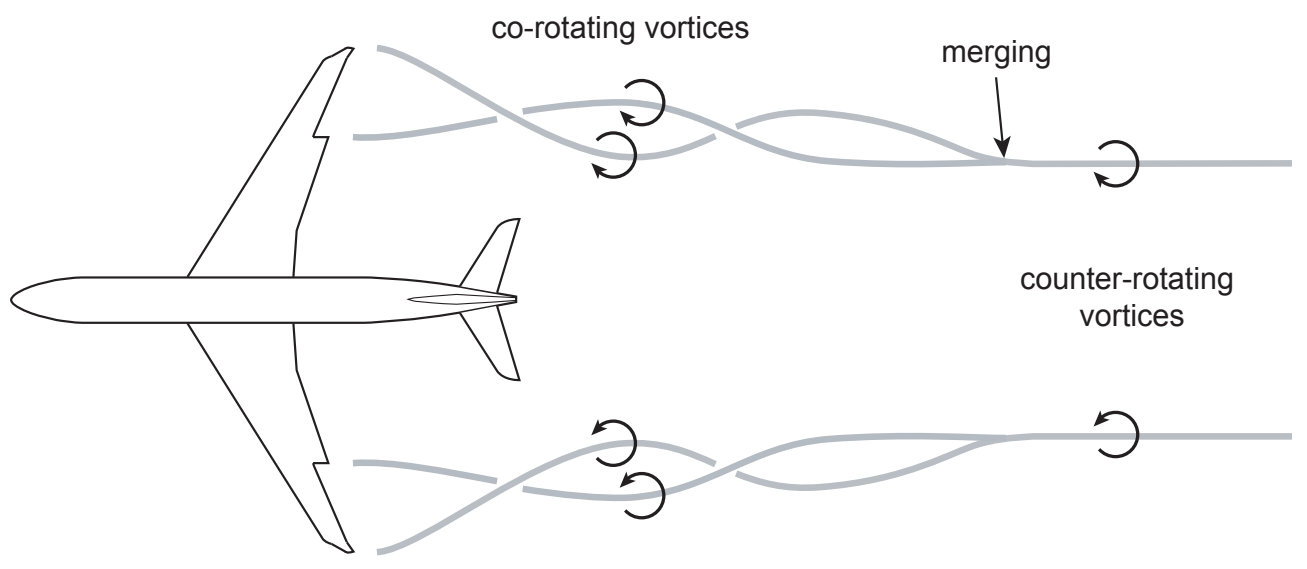

Fig. 1. Schematic of a typical vortex wake of a transport aircraft in high-lift configuration (flaps deflected). The scale in the downstream direction is compressed by a factor between 5 and 10 .

\section{Two-dimensional dynamics}

In this part, we focus on the two-dimensional dynamics of two identical co-rotating vortices having a smooth vorticity distribution. Although such a configuration is a very simplified representation of the near wake of a realistic aircraft, it contains all the ingredients necessary to explore and understand the physics involved in vortex merging. It is also much closer to reality than the constant-vorticity patches, which were frequently used in previous numerical and theoretical studies (see, e.g., Waugh [3], and references therein). Details on the experimental procedures and numerical methods used to obtain the results presented here can be found, e.g., in Meunier et al. [4] and Le Dizès and Verga [5], respectively. Threedimensional effects associated with instabilities will be discussed in detail in section 3 .

\subsection{Interaction of two well-separated vortices}

When two vortices are distant from each other, i.e., when their separation distance $b$ is large compared to their characteristic core radius $a$, the large-scale non-viscous dynamics of the system are well-characterized by a point vortex approach: the two vortices with equal circulation $\Gamma$ remain separated by a constant distance $b_{0}$ and rotate around each other at a constant angular speed

$$
\Omega=\frac{\Gamma}{\pi b_{0}^{2}}
$$

just as if all the vorticity of each vortex was concentrated in the respective vorticity "center of mass", or vorticity centroid.

This large-scale evolution is observed in many experiments, as demonstrated in figure 2(a), 


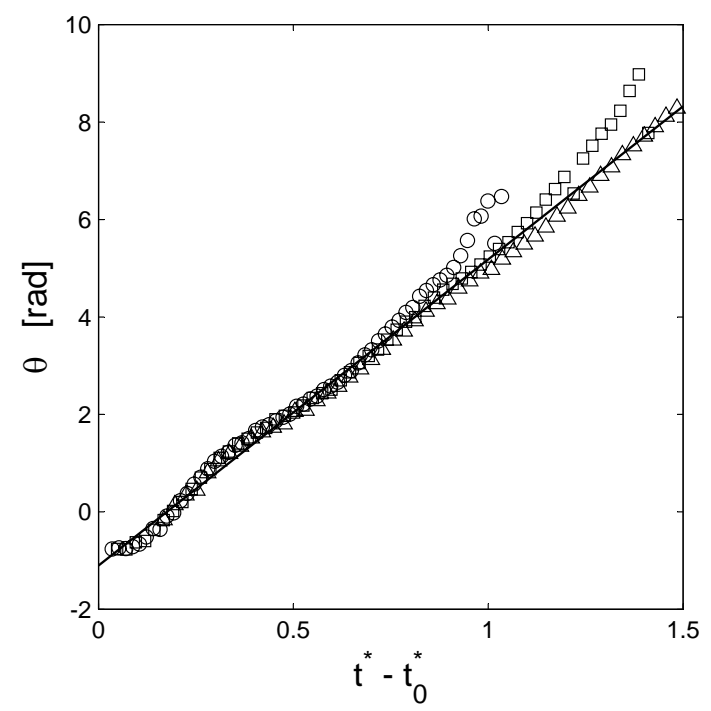

(a)

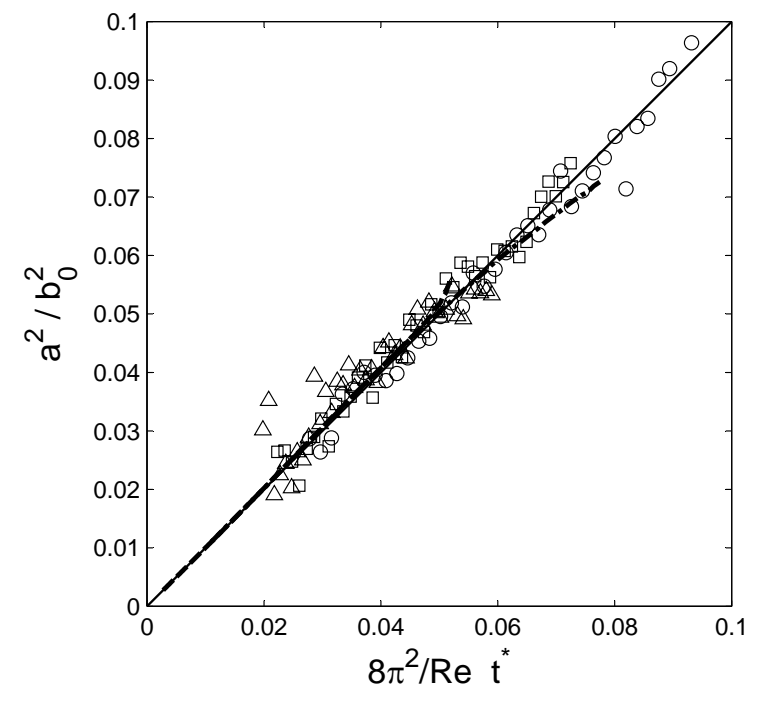

(b)

Fig. 2. Temporal evolution of the orientation (a) and the non-dimensionalized core size (b) of a co-rotating vortex pair. Symbols represent experimental results at $R e=742$ (०), $R e=1506$ ( $\square$ ) and $R e=2258(\triangle)$. Thick lines represent results from direct numerical simulations (DNS) at $R e=500$ (dash-dotted) and $R e=8000$ (dashed). Thin solid lines correspond to the theoretical predictions of Eqs (1) and (4) respectively.

where the angular orientation of the vortex pair is compared with the theoretical prediction (1) for different values of the Reynolds number $R e=\Gamma / \nu$ ( $\nu$ : kinematic viscosity). The period of rotation of the vortex pair is thus a natural characteristic time scale of the system which can be used to define a non-dimensional convective time variable:

$$
t^{*}=t \frac{\Omega}{2 \pi}=\frac{t \Gamma}{2 \pi^{2} b_{0}^{2}} .
$$

While rotating around each other, the vortices spread by viscous diffusion. If the core size of each vortex is defined using the angular momentum $J[4]$ :

$$
a^{2}=\frac{J}{\Gamma}=\frac{\int_{S}\left[\left(x-x_{c}\right)^{2}+\left(y-y_{c}\right)^{2}\right] \omega(x, y) d S}{\Gamma},
$$

where $S$ is a surface containing the vorticity of the vortex, and $\left(x_{c}, y_{c}\right)$ the location of the vorticity centroid, it increases according to the law $a^{2}=4 \nu t$, which can be rewritten in non-dimensional units:

$$
\frac{a^{2}}{b_{0}^{2}}=\frac{8 \pi^{2}}{R e} t^{*}
$$

The temporal evolution of the core size $a$ is plotted in figure 2(b) for experimental and 
numerical results obtained at different Reynolds numbers. It is in excellent agreement with the theoretical prediction (4). This linear evolution allows an unambiguous definition of the origin of time, which is chosen at the time where the backward extrapolated core size vanishes, independently from the actual starting time of the experiments or the simulation.

Le Dizès and Verga [5] have analyzed in detail the viscous evolution of such a vortex pair. They showed that the vorticity profile of each vortex tends to have the same viscous evolution as a single diffusing vortex. Each vortex converges toward a solution close to the Lamb-Oseen vortex whose vorticity and angular velocity profiles are given respectively by:

$$
\omega(r)=\frac{\Gamma}{\pi a^{2}} e^{-r^{2} / a^{2}} \quad ; \quad v_{\theta}(r)=\frac{\Gamma}{2 \pi r}\left(1-e^{-r^{2} / a^{2}}\right) .
$$

The deformation of each vortex, which can be seen, e.g., in figure 4(a), is due to the presence of the other vortex. Each vortex is indeed subjected to the straining field generated by the other vortex and which deforms its streamlines into ellipses. Such deformations were analyzed by Le Dizès [6], who considered an axisymmetric vortex in an external rotating strain field of strain rate $s_{e}$. He observed that, due to the strain-vorticity interaction, the strain rate $s_{i}$ in the vortex center was a complex function of the vorticity profile, the angular rotation rate and the Reynolds number, which could even diverge under certain conditions. For a Gaussian vortex and large Reynolds numbers, a good estimate of the ratio $s_{i} / s_{e}$ versus the angular frequency was found to be given by the relation:

$$
\frac{s_{i}}{s_{e}}=1.5+0.038\left(0.16-\Omega / \omega_{0}\right)^{9 / 5},
$$

where $\Omega / \omega_{0}$ is the ratio of the angular frequency of the strain field and the vorticity in the vortex center. This expression has been compared to data obtained from numerical simulations of vortex pairs [5], by taking for $s_{e}$ the point-vortex estimate $s_{e}=\Gamma / 2 \pi b^{2}$, and for $\Omega / \omega_{0}$, the estimate obtained from (1) and (5): $\Omega / \omega_{0}=a^{2} / b_{0}^{2}$. The results are reproduced in figure 3 ; they show very good agreement. The importance of the inner strain rate $s_{i}$ will be demonstrated in section 3 . We will show that it measures the strength of the three-dimensional instability.

\subsection{Convective merging}

The slow viscous evolution of the two distant vortices is suddenly modified when the vortex core size $a$ exceeds a critical fraction of the separation distance $b$. For the two-vortex system, this critical ratio $(a / b)_{c}$ is always reached, due to the progressive increase of the vortex core size by viscosity. But in other configurations, it could be reached by the action of a background flow that could reduce the separation distance $b$. This situation will again be 


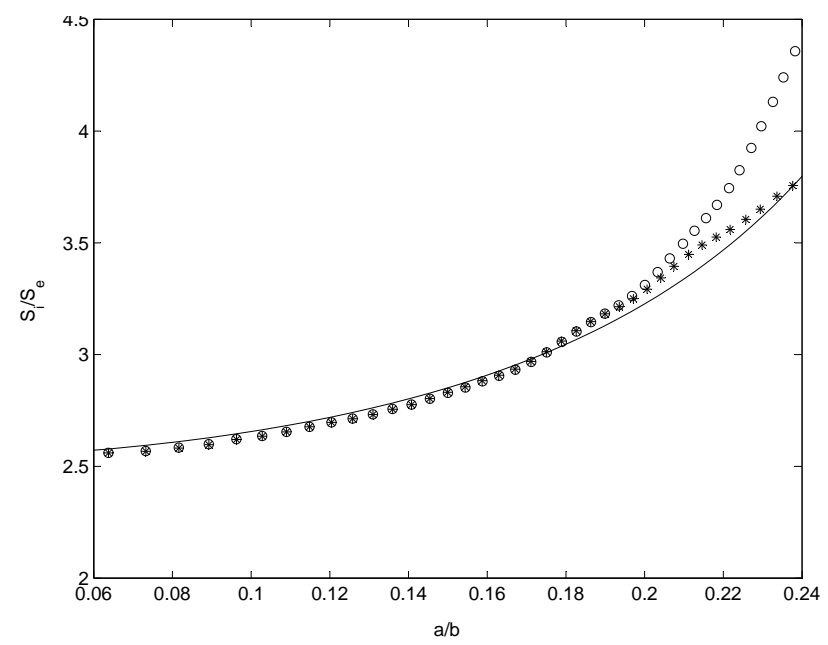

Fig. 3. Ratio of the inner strain $s_{i}$ at the center of the vortex to the outer strain $s_{e}$ induced by the opposite vortex as a function of the dimensionless core size $a / b_{0}$. The solid line corresponds to DNS at $R e=8000$. Symbols correspond to the theoretical prediction of (6) when $\Omega$ is calculated for two vortices separated by $b_{0}(\circ)$ and two vortices separated by the real distance $b(t)$ between the vortices measured in the simulations $(\star)$.

discussed in section 4 . When the critical ratio $(a / b)_{c}$ is exceeded, the two vortices rapidly deform, eject arms of vorticity and merge into a single vortex. Figure 4 presents experimental dye visualisations and numerical vorticity fields of a pair of merging vortices, which rotate anti-clockwise. The two vortices get closer to each other by advection and then coalesce into a single distribution of vorticity by a diffusion process. At the beginning of the merging, two strong filaments of vorticity are ejected and roll-up around the final vortex due to the differential rotation, leading to an axisymmetric vortex at late stages. This merging phenomenon has been extensively studied by different numerical methods $[7,8]$ and was also observed experimentally for geostrophic vortices [9], for starting vortices [4], for aircraft wake vortices $[10,11]$, and in mixing layers [12].

The qualitative phenomenon of merging can be quantitatively studied by measuring the separation distance $b$ between the two maxima of vorticity. It is plotted in figure 5 as a function of convective time for different Reynolds numbers. This evolution allows to define four different stages in the merging process. The first stage corresponds to the quasi-stationary state described in the previous section, for which the separation distance $b$ remains constant, and for which the core size increases by viscous diffusion of vorticity. The duration of this diffusive stage is proportional to the Reynolds number; it can be calculated using (4) as:

$$
\Delta t_{1}^{*}=(a / b)_{c} \frac{R e}{8 \pi^{2}}
$$

Once the critical core size is exceeded, the vortex pair becomes unstable and merges, leading to a rapid decrease of the separation distance. This stage appears to be driven by advection 


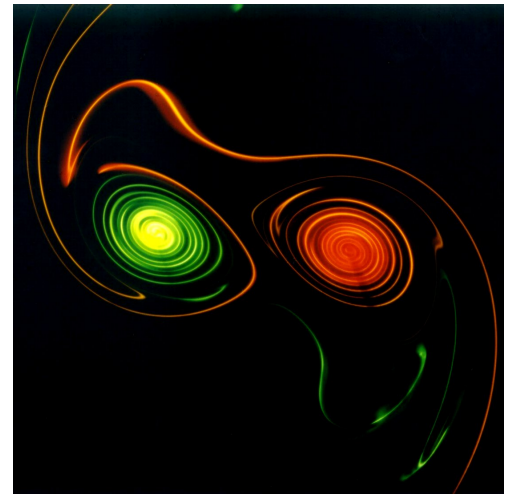

(a)

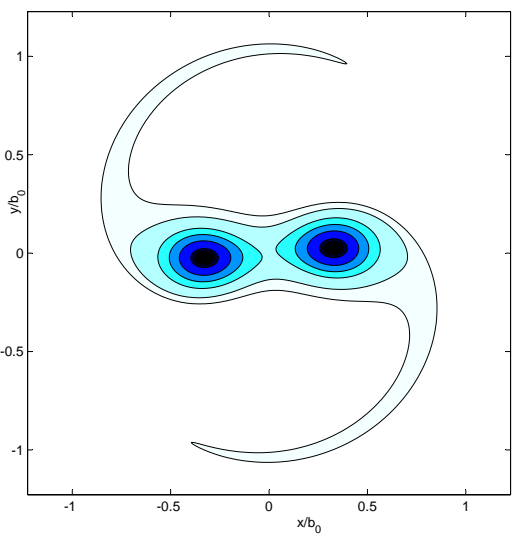

(d)

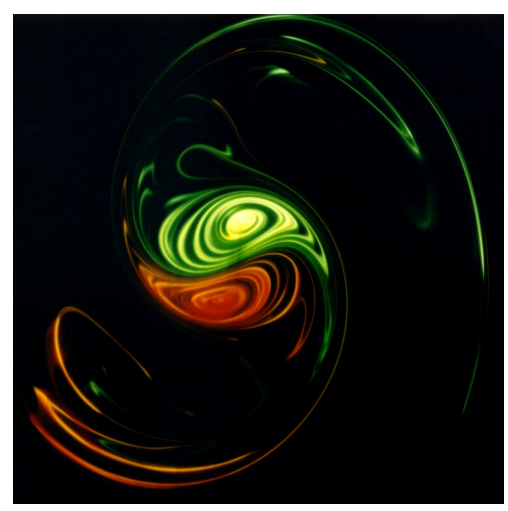

(b)

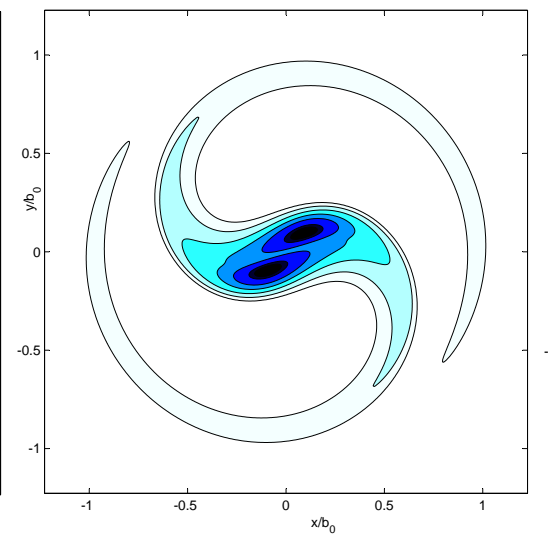

(e)

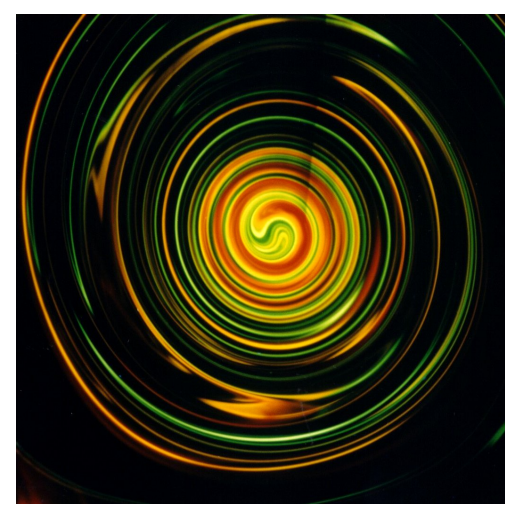

(c)

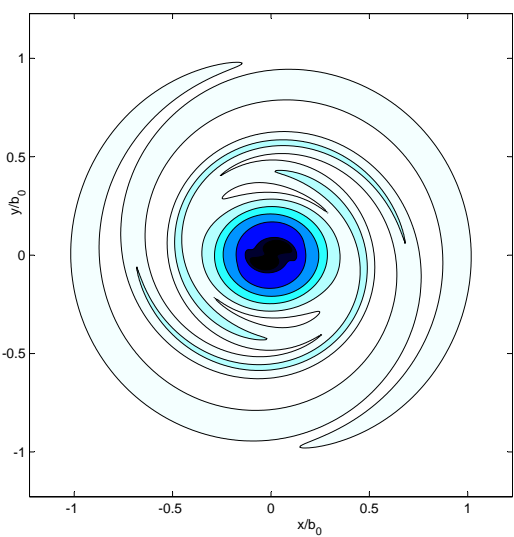

(f)

Fig. 4. (a-c) Cross-cut experimental dye visualizations of two laminar co-rotating vortices, and (d-f) vorticity fields obtained by two-dimensional DNS. The snapshots are taken (a,d) before, (b,e) during and (c,f) after merging.

of vorticity, and its duration $\Delta t_{2}^{*}=0.7$ is thus fairly independent of the Reynolds number. The numerical results in figure 5 show that, at the end of this stage, the merging of the vortices is never complete: the separation distance $b$ does not vanish but remains at a value close to 0.25 . This defines the beginning of a third stage, in which the two vortices are next to each other but still have two separate maxima of vorticity, as can be seen, e.g., in figure 4(e). The diffusion of vorticity, coupled to the rotation of the vortex center (close to a solid body rotation), leads to an axisymmetrization of the vortex center in a time scaling as $R e^{1 / 2}$, as has been explained by Bajer et al. [13] for vorticity perturbations at the center of a vortex. Numerically, the duration of this stage has been found to be:

$$
\Delta t_{3}^{*}=0.0089 R e^{1 / 2}
$$

In the fourth and last stage, the vortex diffuses again due to viscosity and its core size increases with time. This stage will be described in more detail in the next section. 


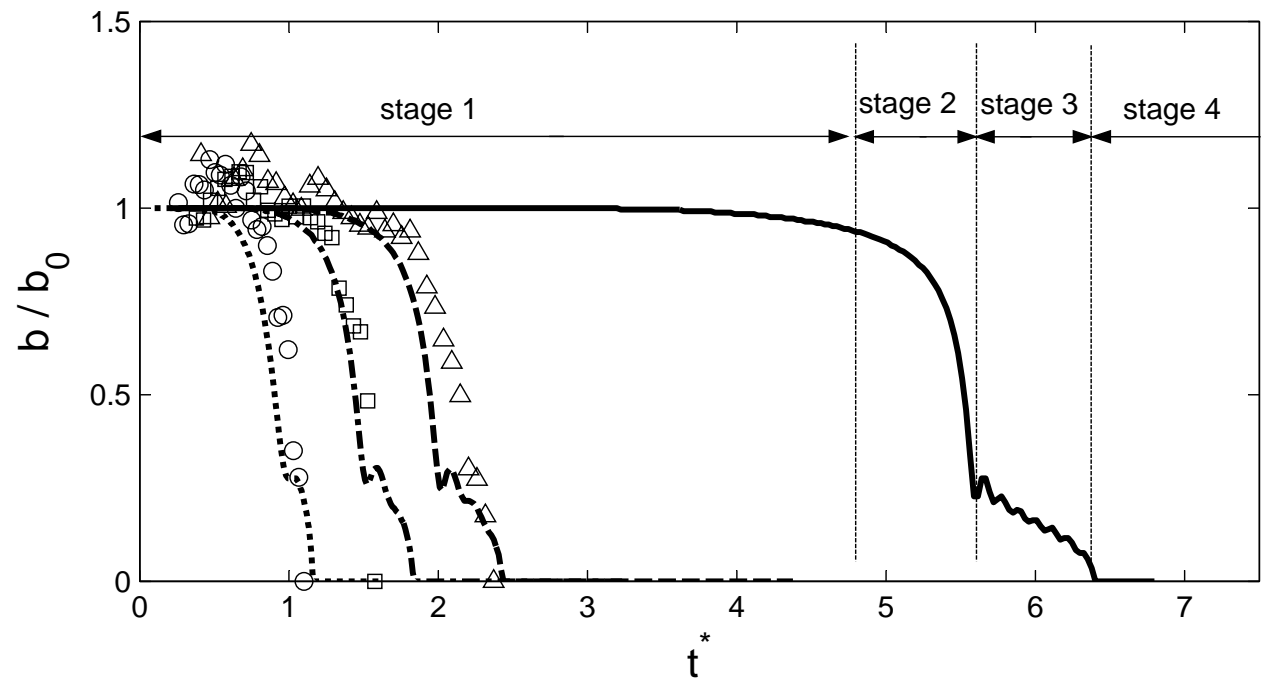

Fig. 5. Separation distance between two merging vortices as a function of the convective time $t^{*}$ for four different Reynolds numbers, obtained experimentally by Particle Image Velocimetry (symbols) and numerically by DNS (lines). $R e=742$ (dotted line, o); $R e=1506$ (dash-dotted line, $\square$ ); $R e=2258$ (dashed line, $\triangle$ ); $R e=8000$ (solid line).

The merging phenomenon highly depends on the critical ratio $(a / b)_{c}$ of core size and separation distance at which it begins. The determination of the critical condition has been the subject of numerous works: through numerical simulations on vortex patches, it was found that $(a / b)_{c}=0.3$ (see Overman and Zabusky [8], Rossow [11], and Dritschel [14,15]), which was confirmed experimentally by Griffiths and Hopfinger [9]. Theoretically, Melander et al. [16] used the elliptic moment model (EMM) to show that the vortex pair is only stable for $(a / b)_{c}<0.326$ and Saffman and Szeto [17] observed the numerical destabilization of two Euler equilibrium solutions for $(a / b)_{c}=0.315$. Recently, Meunier et al. [4] showed experimentally and theoretically using Euler equilibrium solutions that for Gaussian vortices, the criterion is closer to $(a / b)_{c}=0.22-0.24$, which was confirmed experimentally by Cerretelli and Williamson [18] and by direct numerical simulations by Le Dizès and Verga [5]. However, the discrepancy comes from the difference in the vorticity profiles and all the results can be collapsed to $(a / b)_{c}=0.22$ if the core size is defined using (3).

Although the empirical criterion of merging is now known fairly accurately, it is still unclear why exactly the two vortices merge. In order to analyze this issue, it is useful to look at the streamlines in the frame of reference rotating with the vortex pair, because, in the limit of infinite Reynolds numbers, the vorticity contours should be equal to these streamlines 


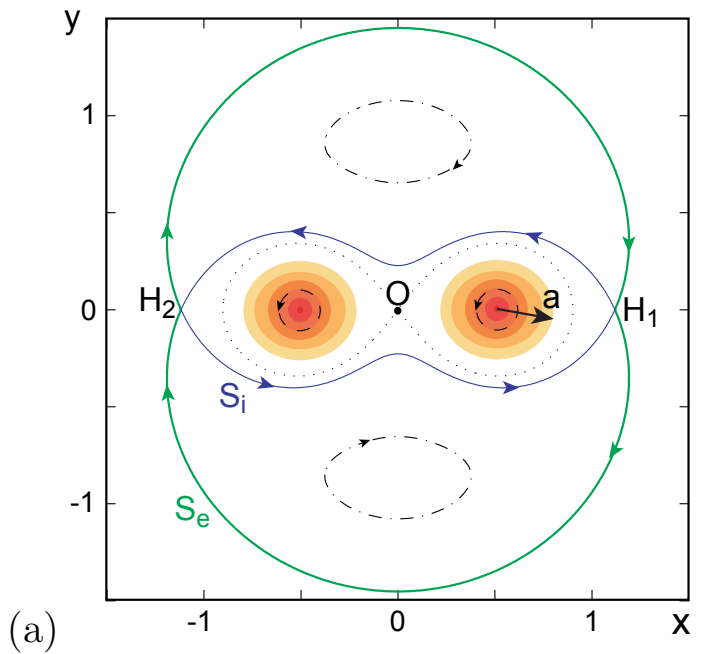

(a)

Fig. 6. Principal streamlines of two co-rotating point vortices in the rotating frame of reference. The vorticity contours (orange colormap) are shown schematically for two vortices whose vorticity (a) remains inside the core of each vortex or (b) diffuses in the exchange band and along the filaments.

(except in the vicinity of hyperbolic points). The characteristic streamlines (separatices) are shown schematically in figure 6 . They divide the plane into four different areas. Inside the dotted streamline, the fluid rotates around only one vortex center. Between the dotted and the blue streamline, the fluid rotates around the two vortices. Between the blue and the green streamline, there are two 'ghost' vortices rotating in the opposite direction. And outside the green streamline, the fluid is almost in solid body rotation.

The theoretical results of Saffman and Szeto [17] and Dritschel [14] tend to prove that each vortex destabilizes before the vorticity touches the center of the pair (named $\mathrm{O}$ in figure 6), as it is the case in figure 6(a). However, Melander et al. [16] showed numerically that it was possible to put some vorticity inside the exchange band without vortex merging. Apparently, the two vortices start moving toward each other when the vorticity reaches the hyperbolic points $H_{1}$ and $H_{2}$, as it is the case in figure 6(b). In this configuration, the vorticity is advected along the green streamlines on figure 6(b) and creates the filaments of vorticity which are observed numerically and experimentally. This argument was used by Meunier [19] to explain the merging process: during the creation of these filaments, they are asymmetric as in figure 4(d) and thus create a velocity field that pushes the two vortices against each other. This hypothesis was verified quantitatively by Cerretelli and Williamson [18]. This argument can be understood in a different way by saying that the ejection of the filaments create a large angular momentum, and thus enforce the vortices to get closer to each other to conserve the angular momentum of the system (which is true for vanishing viscosity).

Using these ideas, a simple model can be constructed, by calculating the angular momentum 
of two vortices of core size $a$ and separated by a distance $b$ : it can be estimated numerically by averaging the vorticity of two circular Gaussian vortices of core size $a$ on the streamlines of two point vortices separated by $b$. This picture is representative of real flows at high Reynolds numbers, since the vorticity is averaged on the whole streamline on a time scaling as $R e^{1 / 3}$ (see Rhines and Young [20], Bernoff and Lingevitch [21]), i.e., much faster than the growth of the core size $a$ which scales as $R e^{1 / 2}$. This is indeed the case in the numerical simulations far from the hyperbolic points. The angular momentum of the system can thus be calculated numerically as a function of $a$ and $b$ :

$$
J=\Gamma b^{2} \widetilde{J}(a / b)
$$

where $\widetilde{J}$ is a universal function shown in figure $7($ a). The angular momentum grows with the ratio $a / b$, even faster than for two vortices whose vorticity distribution is not averaged on the streamlines (shown as a thin solid line in figure 7(a)). This comes from the fact that the streamline averaging creates the vorticity filaments, which increases the angular momentum. To reach a theoretical prediction of the separation distance $b$, the expressions of the core size $a$ given by Eq. (4) and the expression of the angular momentum $J$ as a function of time (given by Dritschel [14] for a two-dimensional flow):

$$
J=\Gamma b_{0}^{2} / 2+8 \nu \Gamma t
$$

can be introduced into Eq.(9), leading to the prediction of $b(t)$. The result is plotted in figure 7 and compared to the numerical results. This simple model predicts relatively well the time at which the separation distance starts to drop. However, it does not predict a total merging of the vortices, but only explains why they come closer to each other up to $80 \%$ of the initial distance. In order to fully explain the merging process, some other phenomenon, probably associated with a convective instability of the vortex system, has to be invoked. However, a theoretical prediction and description of such an instability are still missing so far.

\subsection{Characteristics of the final vortex}

After the convective phase of merging, the resulting vortex becomes axisymmetric on a time scale $R e^{1 / 2}$, which is small compared to the viscous time scale. The merging and axisymmetrization processes can therefore be considered as instantaneous on such a viscous time scale. Our objective is here to characterize the final axisymmetric vortex which results from these processes.

Since the final vorticity profile will turn out to be non-Gaussian, it is convenient to define the vortex core size as the radius $a_{\max }$ at which the azimuthal velocity is maximum, divided by 1.12 (equivalent to the core size $a$ for a Gaussian vortex). Using this definition, the core 

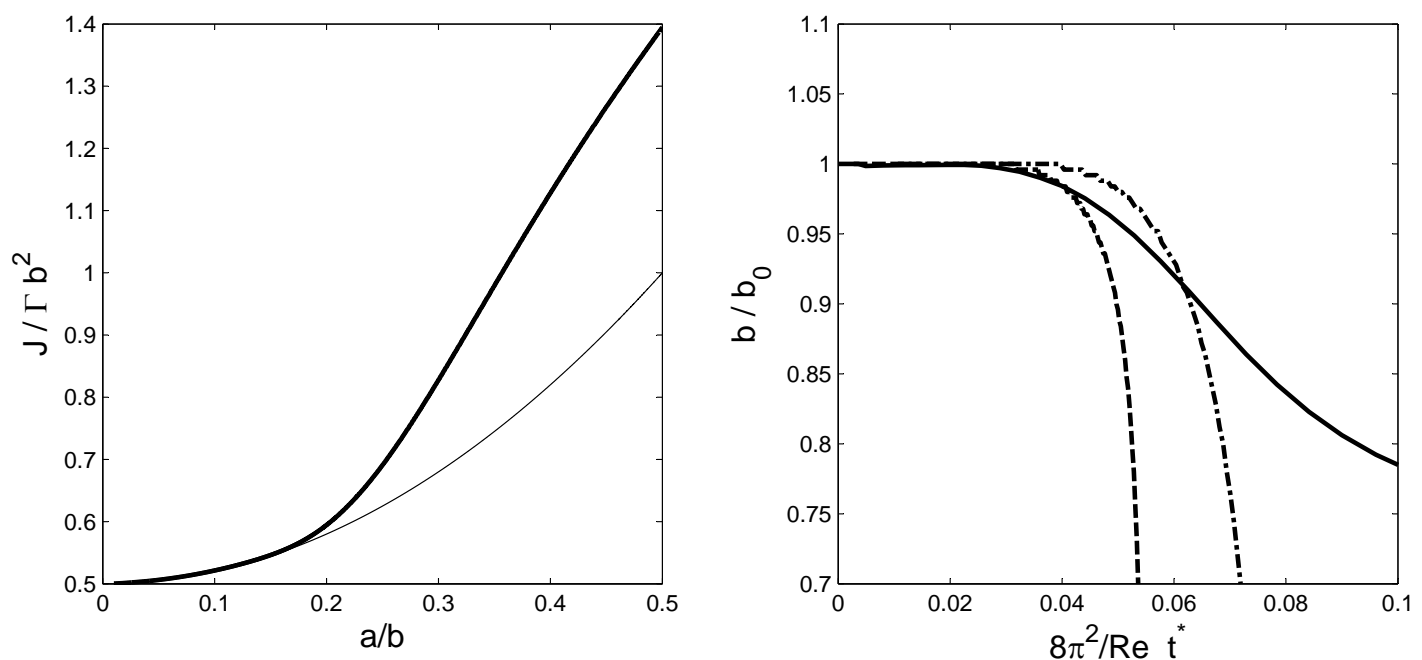

Fig. 7. (a) Rescaled angular momentum $J$ of two vortices of core size $a$ separated by $b$, which are axisymmetric (thin line) and whose vorticity has been averaged numerically along the streamlines of two point vortices (thick line). (b) Theoretical prediction of the separation distance as a function of the viscous time (solid line), compared to the numerical results (dashed line: $R e=8000$ and dash-dotted line: $R e=1506)$.

size can be measured before and after merging; this is plotted in figure 8 for three different Reynolds numbers as symbols and dashed lines. The square of the core size increases before and after merging linearly in time with the same slope $4 \nu$ (obtained for Gaussian vortices) and jumps abruptly during the merging stage. Although this stage lasts longer at low Reynolds numbers, a universal behavior is observed: the square of the core size $a^{2}$ increases by a factor 1.5 when measured at the end of the merging stage. This behavior is more universal than the increase of a factor 2 given in the paper by Meunier \& Leweke [22], where it was measured at the beginning of the merging stage.

A prediction of a similar core size ratio had been made by Carnevale et al. [23], assuming that the maximum vorticity $\omega_{\max }$ and the energy (scaling as $\omega_{\max }^{2} a^{4}$ ) are conserved: the energy of the final vortex is twice the energy of each initial vortex, and the square of the core size $a^{2}$ thus increases of a factor $\sqrt{2}$ during the merging, which is close to the experimental and numerical value of 1.5 .

However, this theory is in contradiction with the conservation of the circulation and the angular momentum. Indeed, if the core size is defined as in Eq. (3) using the angular momentum $J$ and total circulation $\Gamma_{\text {tot }}$ (shown as a thick solid line in figure 8), the core size increases by a much larger factor than when it is defined using $a_{\max }$. This ratio can be predicted theoretically using the expression of the angular momentum given in (10) (plotted as a thin solid line in figure 8). The difference between these two measurements 


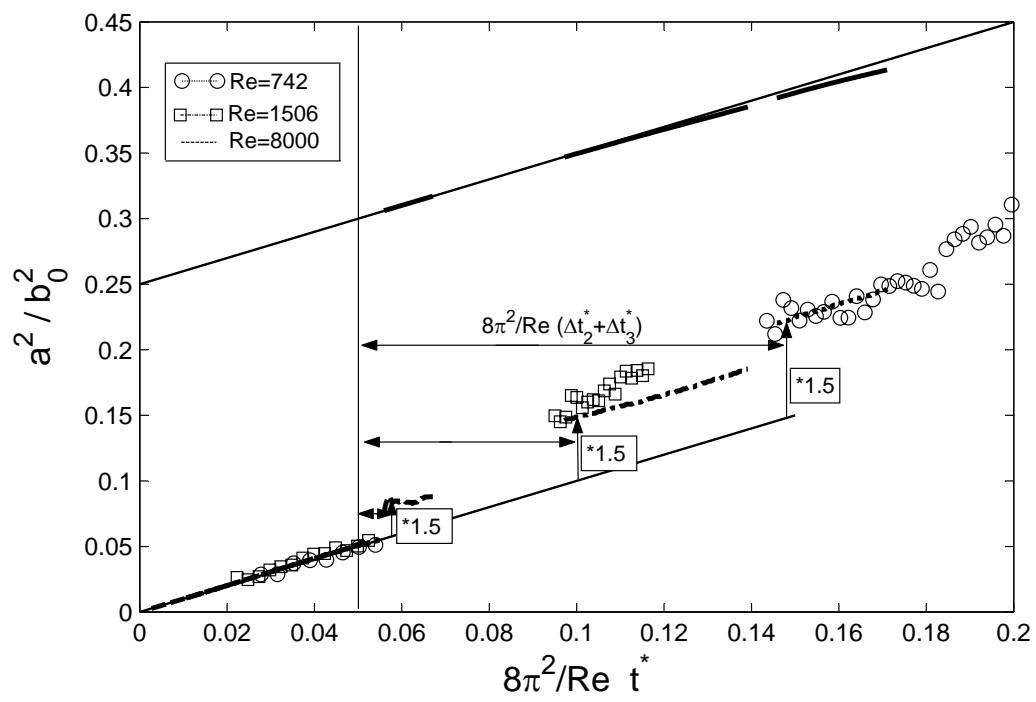

Fig. 8. Dimensionless core size $a / b_{0}$ of the initial and final vortices for various experimental (symbols) and numerical (lines) results. The core size is defined as $a_{\max } / 1.12$ for the dashed lines and for the symbols, and it is defined as $\sqrt{J / \Gamma}$ for the thick solid lines. The thin solid lines are predictions for Gaussian vortices.

comes from the fact that the velocity profile is not Gaussian after merging: the final vortex contains a core with an intense vorticity, surrounded by a background of low vorticity far from the center coming from the ejected filaments. The theory of Carnevale et al. [23] predicts accurately $a_{\max }$ (since the core contains most of the energy), and the theory using the conservation of the angular momentum is linked to the filaments (since they contain most of the angular momentum).

As a consequence, none of the previous theories predicts in a satisfactory way the final velocity profile, which is plotted in figure 9. The theory by Carnevale et al. (shown as a dotted line on figure 9) predicts accurately the velocity and the circulation only inside the core of the final vortex (since most of the energy is located in the vortex core). On the contrary, the theory using the conservation of the angular momentum (shown as a dashed line on figure 9) predicts accurately the velocity and circulation in the filaments, i.e. far from the center, but fails in the vortex core.

An alternative theory is thus needed to describe accurately the entire velocity profile of the final vortex. Since the core and the filaments are created by two different mechanisms (fusion of two cores and ejection of vorticity), we assume that they can be modeled separately as Gaussian vortices with different core sizes and circulations. We thus seek the vorticity profile 

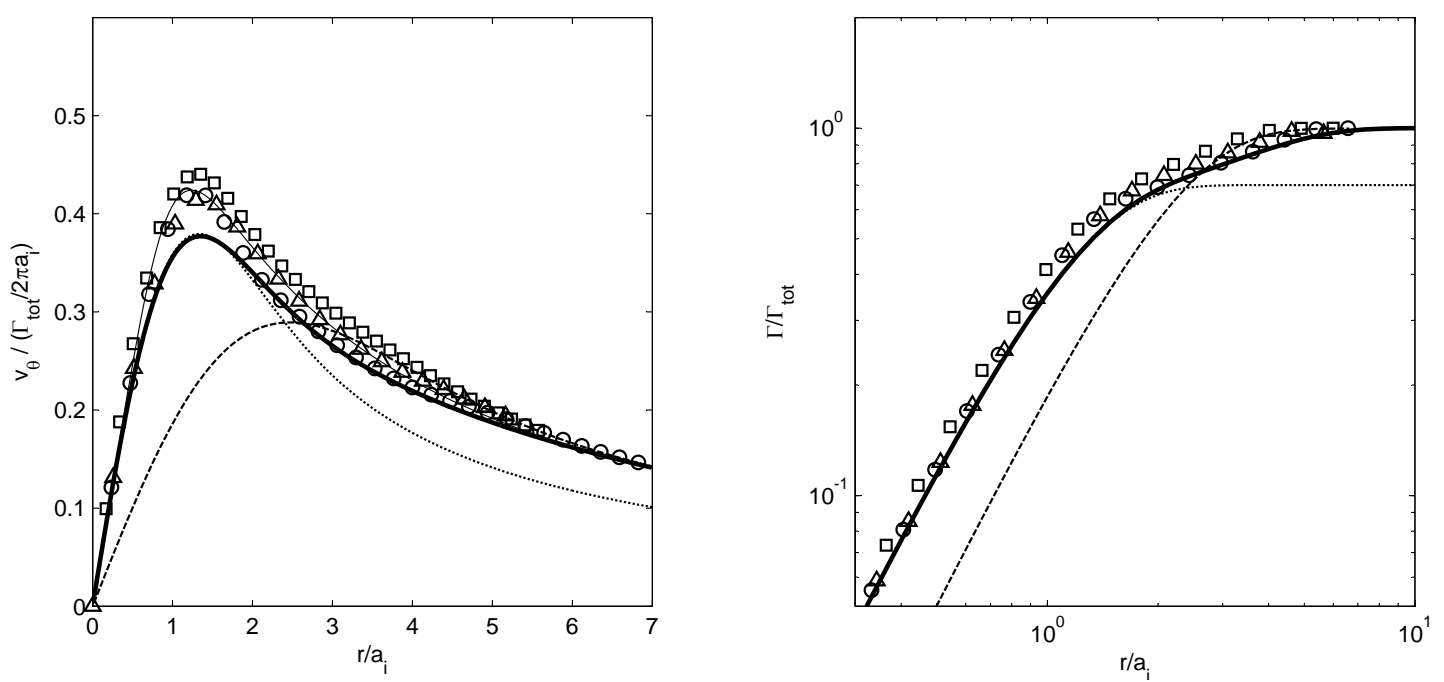

Fig. 9. (a) Velocity and (b) circulation profiles of the final vortex, rescaled using the total circulation $\Gamma_{\text {tot }}$ of the flow and the core size of the initial vortex before merging $a_{i}=\sqrt{4 \nu t}$. The symbols show results from numerical simulations at $R e=8000$ (०) and $R e=1506$ ( $\square$ ) and from experiments at $R e=1506(\triangle)$. The dotted line corresponds to a Gaussian vortex with a core size predicted by the theory of Carnevale et al. [23] and the dashed line to a Gaussian vortex with a core size predicted using the conservation of the angular momentum. The thick solid line corresponds to the theory using two concentric vortices with different core sizes given by (11). The thin solid lines is a fit to a two-scale model defined by Fabre and Jaquin [24], given by (16).

of the final vortex as:

$$
\omega=\frac{\Gamma_{c}}{\pi a_{c}^{2}} e^{-r^{2} / a_{c}^{2}}+\frac{\Gamma_{f}}{\pi a_{f}^{2}} e^{-r^{2} / a_{f}^{2}}
$$

where the four variables are: $\left(\Gamma_{c}, a_{c}\right)$ the circulation and size of the core, and $\left(\Gamma_{\mathrm{fil}}, a_{\mathrm{fil}}\right)$ the circulation and size of the wrapped filaments. We now look for a solution which has the same energy, maximum vorticity, angular momentum and circulation as the initial pair of Gaussian vortices of circulation $\Gamma$, initial core size $a_{i}$, and separated by $b$. The conservation of the total circulation writes:

$$
\Gamma_{\text {tot }}=\Gamma_{c}+\Gamma_{f}=2 \Gamma \text {. }
$$

The conservation of maximum vorticity means:

$$
\frac{\Gamma_{c}}{\pi a_{c}^{2}}+\frac{\Gamma_{f}}{\pi a_{f}^{2}}=\frac{\Gamma}{\pi a_{i}^{2}} .
$$

The conservation of the angular momentum leads to:

$$
\Gamma_{c} a_{c}^{2}+\Gamma_{f} a_{f}^{2}=2 \Gamma a_{i}^{2}+\Gamma b^{2} / 2 .
$$


After a straightforward calculation (see Appendix), the conservation of the excess energy leads to:

$$
\Gamma_{c}^{2}\left[-C-\ln \left(\frac{a_{c}}{a_{i}}\right)\right]+\Gamma_{f}^{2}\left[-C-\ln \left(\frac{a_{f}}{a_{i}}\right)\right]+2 \Gamma_{c} \Gamma_{f}\left[K_{\mathrm{conc}}\left(\frac{a_{c}}{a_{f}}\right)-\ln \left(\frac{a_{c}}{a_{i}}\right)\right]=-2 \Gamma^{2} C-2 \Gamma^{2} K_{\mathrm{sep}}\left(\frac{b}{a_{i}}\right)
$$

Here, $\mathrm{C}$ is a constant, $K_{\text {conc }}$ is the mutual energy of two concentric vortices and $K_{\text {sep }}$ is the mutual energy of two vortices separated by $b$, given in the appendix. For the critical ratio of core size and separation distance $(a / b)_{c}=0.22$, the four preceding equations have a solution: $a_{c}=1.14 a_{i}, \Gamma_{c}=1.22 \Gamma, a_{f}=3.71 a_{i}$ and $\Gamma_{f}=0.78 \Gamma$. For these parameters, the square of the core size (defined as $\left.\left(a_{\max } / 1.12\right)^{2}\right)$ increases of a factor 1.46, in excellent agreement with the measured value of 1.5 (see figure 8 ). This corresponding velocity profile is plotted in figure 9 and is very close to the experimental and numerical velocity profiles both in the core of the vortex and in the far field. The velocity is only $10 \%$ smaller than the measured value at the maximum. This is due to a slight loss of energy during the merging stage, and a better agreement can be obtained (within the noise in the measurements) by assuming an energy loss of $10 \%$ during the merging stage. The agreement is even better for the circulation profiles. Using this vortex model, the evolution of the final vortex is straightforward since the velocity profile corresponding to Eq. (11) is a solution of the Navier-Stokes equations, if the squares of the core sizes increase linearly in time.

The final velocity profile can be also fitted by a two-scale model described by Fabre and Jacquin [24], which contains three fitting parameters (the circulation $\Gamma_{\text {tot }}$ being equal to $2 \Gamma)$. The best fit, shown in figure 9 as a thin solid line, is given by:

$$
v_{\theta}(r)=\frac{\left(a_{2} / a_{1}\right)^{\alpha} \Gamma_{\text {tot }} /\left(2 \pi a_{1} a_{2}\right)}{\left[1+\left(r / a_{1}\right)^{4}\right]^{\frac{1+\alpha}{4}}\left[1+\left(r / a_{2}\right)^{4}\right]^{\frac{1-\alpha}{4}}}
$$

with $\alpha=2 / 3, a_{1}=1.12 a_{i}$ and $a_{2}=4.5 a_{i}$. This vortex model is useful since it was shown to be characteristic of airplane vortices, and was analyzed in detail with respect to three-dimensional instabilities [24].

To conclude, in this section, we have described the flow of two co-rotating vortices before merging, we have determined the empirical criterion for merging and we have also characterized the final vortex. However, these analyses have been made for a two-dimensional flow, which is valid representation of real flows only at low Reynolds numbers $(R e<2000)$. When the Reynolds number increases, the flow can become three-dimensional through instabilities and turbulence, which can highly modify the merging phenomenon. The merging at high Reynolds numbers will be presented in the following chapter. 


\section{Three-dimensional dynamics}

For large Reynolds numbers, the two-dimensional flow obtained by the interaction of two co-rotating vortices before their merging forms a quasi-steady solution in the frame rotating with the vortex pair. In view of the complex streamline pattern (figure 6a), it is natural to address the stability characteristics of such a $2 \mathrm{D}$ solution, with respect to three-dimensional perturbations.

For two counter-rotating vortices, three-dimensional stability studies were started in the 1970's. Crow [25] showed that a pair of counter-rotating vortices is unstable with respect to a long-wavelength instability. This instability which is now known to play an important role in the dynamics of the far wake, leads to the reorganization of the vortices into vortex rings. Leweke and Williamson [26] demonstrated that this instability can be mixed with a short-wavelength instability associated with the elliptic character of the streamlines in the vortices.

When the vortices are co-rotating, Jimenez [27] proved that the long-wavelength instability obtained by Crow could not be active. By contrast, the short-wavelength elliptic instability is present, as first evidenced by Meunier and Leweke [28]. The following section will be concerned with the description of its characteristics in a system of two co-rotating Gaussian vortices.

The streamline pattern also exhibits hyperbolic points. Near these stationary points, vorticity can be locally increased by stretching. In mixing layers and wakes, during their threedimensional transition, this hyperbolic stretching mechanism is known to participate in the formation of ribs aligned along the stretching direction between successive vortices. For two distinct vortices, as those considered in the previous section, there exists no evidence of such ribs. Yet, it cannot be discarded in more realistic configurations, notably when a vortex sheet is still present between the vortices, that the local growth near hyperbolic points could contribute to the global growth of a three-dimensional instability modes, in particular those associated with the elliptic instability.

\subsection{Elliptic instability}

In this section, a short description of the elliptic instability which develops in a co-rotating vortex pair is given. A more detailed account can be found in Le Dizès and Laporte [29] for the theoretical aspects and in Meunier and Leweke [22] for the experimental results.

Experiments and direct numerical simulations show that, when the Reynolds number ex- 


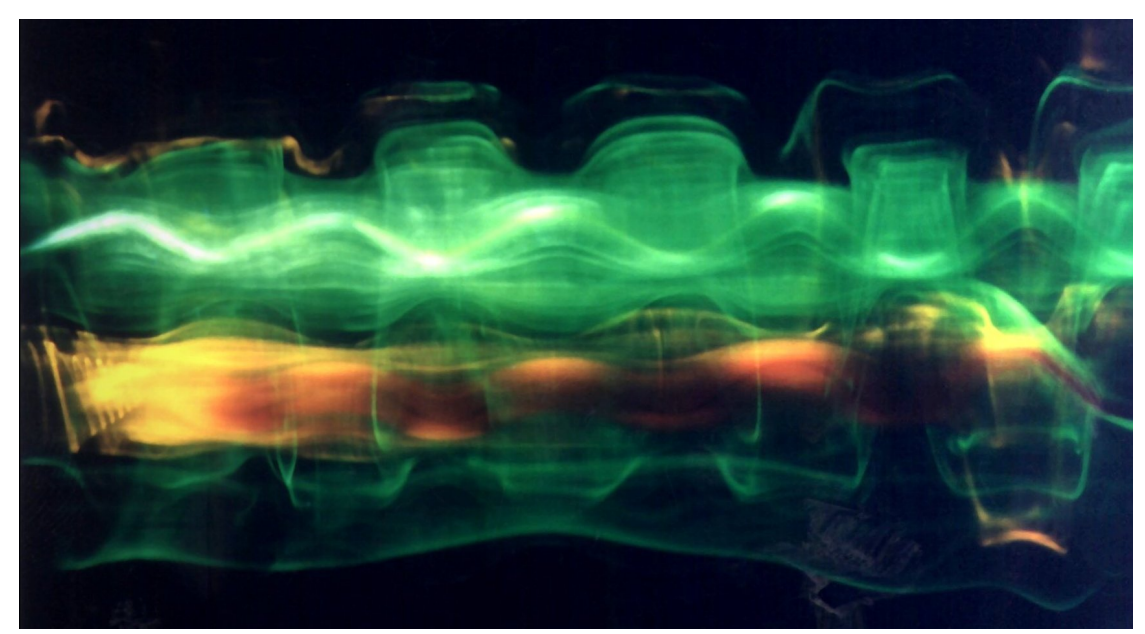

(a)

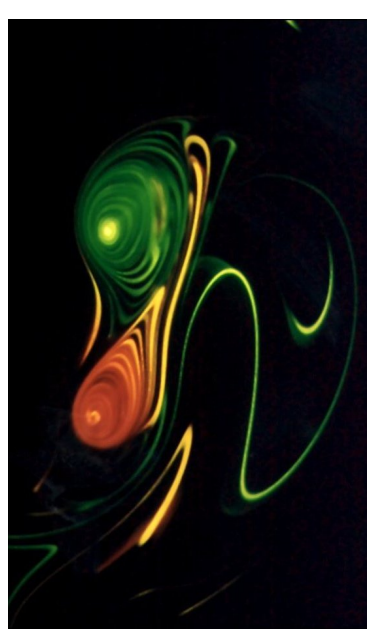

(b)

Fig. 10. Elliptic instability of a co-rotating vortex pair. Dye visualizations from experiments in (a) a side-view and (b) a cross-cut section.

ceeds approximatively $R e \approx 2000$, a three-dimensional perturbation grows spontaneously in the vortices before their merging. This instability, which is illustrated in figures 10(a) and 11(a), is characterised by a sinuous deformation of each vortex in two parallel planes. Moreover, the perturbation is found not to propagate and to have a well-defined wavelength which scales with the core size. Its transverse structure is such that it breaks the mirror symmetry of the two-dimensional system (figures 10b and 11c). Figures 12(a,b) show the axial vorticity of the perturbation alone obtained in experiments and in numerical simulations, respectively. It has a dipolar shape oriented at approximatively $45^{\circ}$ with respect to the line connecting both vortices.

These instability characteristics are typical of what is now called an elliptic instability (see the review of Kerswell [30]). It has also been observed in counter-rotating vortices [26], in vortex rings [31], in elliptically deformed cylinders [32], but is also expected to take part in the three-dimensional destabilization of parallel shear flows [33] and wakes [34]. The common characteristic of all these flows is the presence of elliptically deformed vortices. The instability mechanism was understood by Pierrehumbert [35] and Bayly [36], who demonstrated that any elliptic uniform flow was generically unstable with respect to short-wavelength perturbations in a non-viscous flow. In the present case, the base flow in the frame co-rotating with the vortex pair is elliptic near the center of each vortex. The base flow streamfunction can be written in polar coordinates centered on one vortex, near its center, as

$$
\Psi \approx-(\mu-\Omega) \frac{r^{2}}{2}-s_{i} \frac{r^{2}}{2} \cos (2 \theta+\phi)
$$

where $\mu=2 \omega_{0}$ and $s_{i}$ are the angular velocity and the inner strain rate in the vortex center, and $\Omega$ is given by (1). The phase angle is such that $\phi \approx \pi / 4$ if $\theta=0$ corresponds to the 


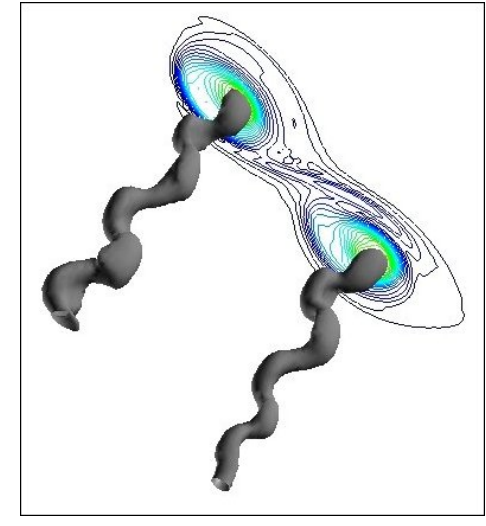

(a)

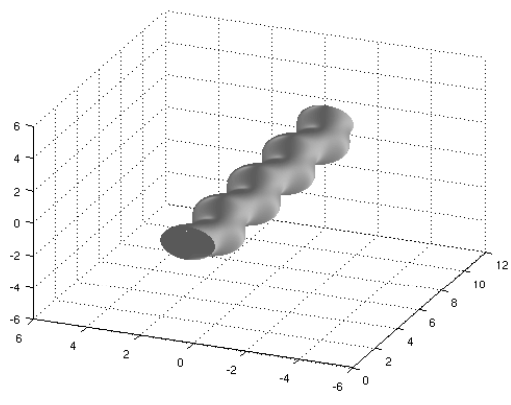

(b)

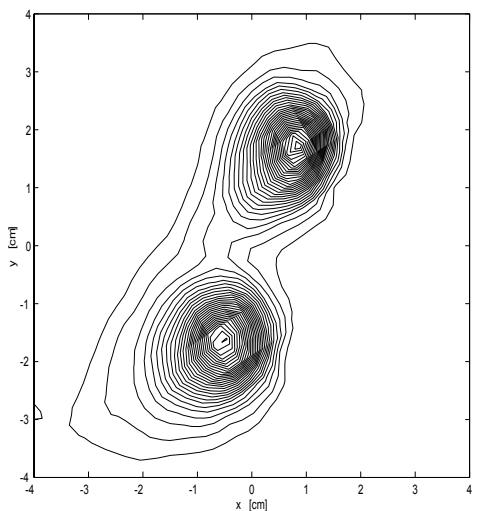

(c)

Fig. 11. Elliptic instability characteristics. Perspective view of the vorticity isosurfaces obtained by (a): Direct Numerical Simulations of co-rotating vortices, and (b): the theory in a single vortex as combination of the underlying vortex with two Kelvin waves $m=-1$ and $m=1$. (c): Cross-cut axial vorticity obtained from experiments with $R e=3450$.

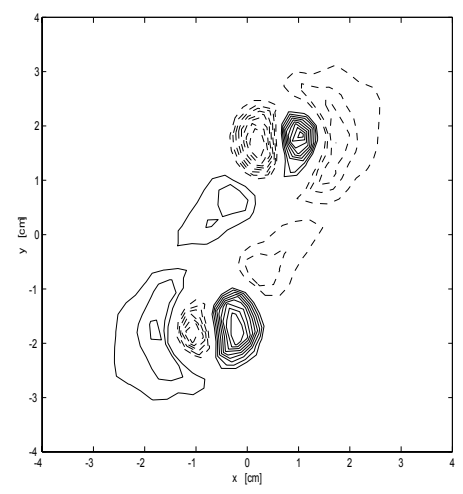

(a)

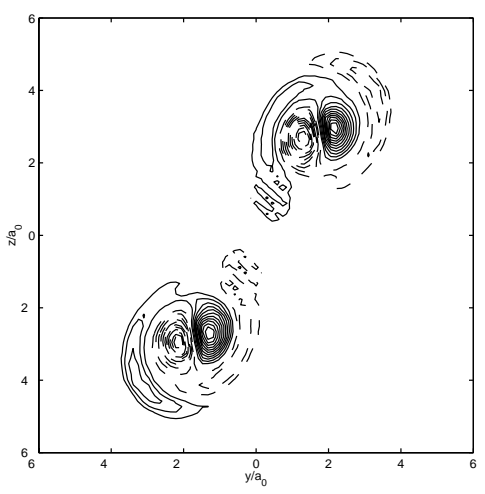

(b)

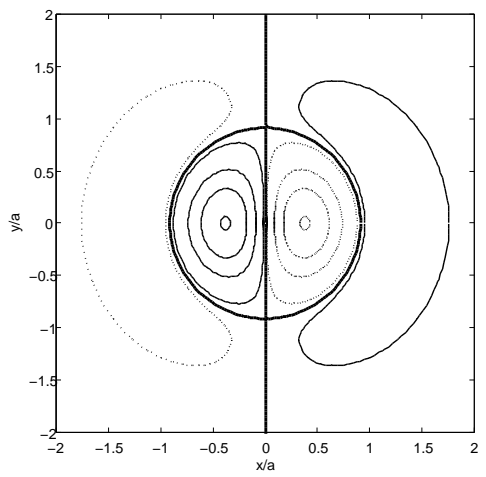

(c)

Fig. 12. Axial vorticity of the elliptic instability mode. (a): Experimental results for $R e=3450$; (b): Numerical results using Large-Eddy Simulations (LES) for $R e=10^{5}$ [37]; (c): Theoretical prediction in a single vortex in which the perturbation is a combination of Kelvin waves $m=1$ and $m=-1$.

direction of the line connecting both vortex centers.

The streamlines defined by (17) are ellipses of eccentricity $\varepsilon=s_{i} /(\mu-\Omega)$. If one considers local perturbations near the vortex center, where the base flow is defined by (17), a general stability analysis can be performed, as shown by Bayly [36] and Lifschitz and Hameiri [38]. For small elliptic deformation $(\varepsilon \ll 1)$, an asymptotic estimate for the growth rate of the three-dimensional local plane waves

$$
\mathbf{u}=\mathbf{u}_{\mathbf{o}} e^{i \mathbf{k}(t) \cdot \mathbf{x}-i \omega t}
$$


can be obtained [39] as

$$
\sigma^{(\text {local })}=\sqrt{\left(\frac{3 \mu-\Omega}{4 \mu}\right)^{4} s_{i}^{2}-(\mu-\Omega-2 \mu \cos \xi)^{2}}-\frac{k_{z}^{2}}{R e \cos ^{2} \xi},
$$

where $\xi$ is the constant angle between the wavevector $\mathbf{k}$ and the vortex axis. This local growth rate estimate is maximum when $\cos \xi \equiv k_{z} /|\mathbf{k}|=(\mu-\Omega) /(2 \mu)$.

Le Dizès and Laporte [29] used the above formula for the growth rate of the elliptic instability for two co-rotating Gaussian vortices. They were able to evaluate the local wavevector angle $\xi$ of the perturbation by performing a global analysis of the elliptical instability. Such an analysis was first performed by Moore and Saffman [40] and Tsai \& Widnall [41]. The idea is to take into account the complete structure of the vortex. Each vortex possesses, when not deformed by the presence of the other vortex, an infinite number of normal modes called Kelvin waves of the form

$$
\mathbf{u}=\mathbf{u}_{\mathbf{K}}(r) e^{i k_{z} z+i m \theta-i \omega t},
$$

whose axial wavenumber $k_{z}$, azimuthal wavenumber $m$, and frequency $\omega$ satisfy a dispersion relation $D\left(k_{z}, m, \omega\right)=0$. This dispersion relation depends on the vorticity profile of the vortex. A description of those waves can been found in [42] for the Rankine vortex and in Fabre et al. [43] for the Lamb-Oseen vortex. For these two types of vortices, none of these waves are unstable by itself. However, they can be resonantly coupled with each other when one considers the elliptic deformation field induced by the other vortex. Indeed, such a field can be written, in a fixed frame centered on one vortex, as a velocity correction of the form

$$
\mathbf{U}_{\mathbf{1}}=\mathbf{V}_{\mathbf{1}}(r) e^{2 i(\theta-\Omega t)}
$$

It can therefore couple two neutral Kelvin waves $\left(k_{z_{1}}, m_{1}, \omega_{1}\right)$ and $\left(k_{z_{2}}, m_{2}, \omega_{2}\right)$, if they satisfy the conditions of resonance

$$
k_{z_{1}}=k_{z_{2}} ; m_{1}=m_{2}+2 ; \omega_{1}=\omega_{2}+2 \Omega \text {. }
$$

In this description, the elliptic instability is thus interpreted as a resonance phenomenon of Kelvin waves with the straining field. In most cases, there exist several pairs of Kelvin waves satisfying (22), but these configurations are not excited with the same growth rate. It turns out that, for vortices such as the Lamb-Oseen vortex, the most unstable configurations satisfy a peculiar property near the vortex center: they locally correspond to a combination of local plane wave of the form (18) if their frequency satisfies the additional conditions

$$
\omega_{1}=\left(m_{1}-1\right) \mu+\Omega ; \omega_{2}=\left(m_{2}+1\right) \mu-\Omega .
$$

These two conditions are satisfied by the two symmetric and resonant Kelvin waves $m_{1}=1$, $\omega_{1}=\Omega$ and $m_{2}=-1, \omega_{2}=-\Omega$ for a discrete number of wavenumbers $k_{z}^{(n)}(\Omega)$. The 


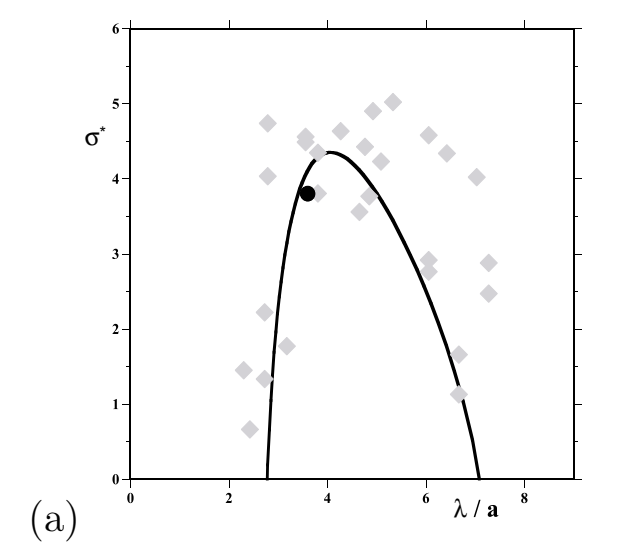

(b)

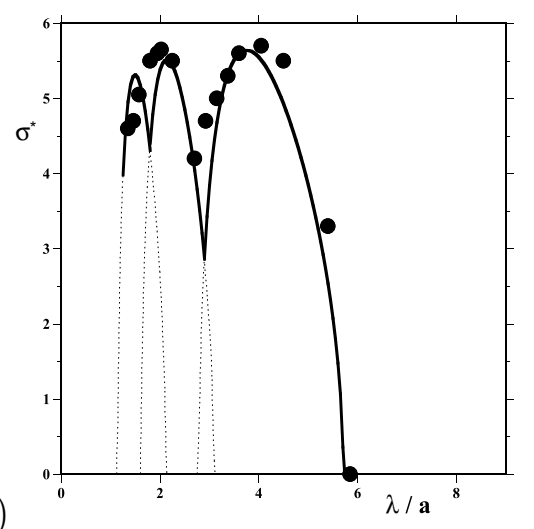

Fig. 13. Growth rate of the elliptic instability as a function of the axial wavelength (a) at $R e=2700$ and $a / b=0.220$, and (b) at $R e=10^{5}$ and $a / b=0.205$. Growth rate is non-dimensionalized by the rotation period of the pair. Lines correspond to theory, black symbols to DNS/LES and gray symbols to experiments. From [37].

combination of these two waves forms a sinusoidal deformation in a plane rotating at the angular velocity of the vortex pair. Figure 12(c) shows the axial vorticity associated with the combination of the first two resonating waves. It is in qualitative agreement with the experimental and numerical results shown in figures $12(\mathrm{a})$ and $12(\mathrm{~b})$, respectively. Figure 11(b) shows a surface of constant axial vorticity of the total flow composed of the underlying vortex and the two resonant Kelvin waves. The same undulation as observed in figure 11(a) is obtained.

If one assumes that the growth of the two resonant waves is dominated by the local growth near the vortex center, formula (19) can be used as soon as a connection between $\cos \xi$ and the dispersion relation of the Kelvin modes has been made. This is possible by expressing the resonant Kelvin waves near the origin as local plane waves, which gives, for the mode $m=1$, the relation

$$
\cos \xi^{(n)}=\frac{1}{2}-\frac{\omega^{(n)}\left(k_{z}\right)}{2 \mu},
$$

where $\omega^{(n)}\left(k_{z}\right)$ is the $n$th eigenfrequency of the Kelvin wave $m=1$. The number $n$ denotes the index of the Kelvin wave. It can be associated with the number of radial oscillations of the velocity components of the Kelvin wave. The larger the index, the more oscillatory the Kelvin waves structure is. As the maximum growth rate is obtained for small values of $\omega^{(n)} /(2 \mu)$ [close to $\Omega /(2 \mu)$ as prescribed by $(23)$ ], a good estimate can be obtained by a linear fit of the different branches for small frequencies. For the Lamb-Oseen vortex, we obtain the following relations

$$
\cos \xi^{(n)}=\frac{1}{2}-\frac{2.26+1.69 n-k_{z} a}{14.8+9 n} ; n=0,1,2, \ldots
$$


If we non-dimensionalize the instability growth rate (19) by the convective time scale $2 \pi / \Omega$, we finally obtain an expression of the form

$$
\sigma^{*}=\pi \sqrt{\left[\frac{3}{4}-\frac{1}{2}\left(\frac{a}{b}\right)^{2}\right]^{4}\left(\frac{s_{i}}{s_{e}}\right)^{2}-4\left(\frac{b}{a}\right)^{4}\left[\frac{1}{2}-\left(\frac{a}{b}\right)^{2}-\cos \xi^{(n)}\right]^{2}}-\frac{2 \pi^{2}\left(k_{z} b\right)^{2}}{R e \cos ^{2} \xi^{(n)}},
$$

which, for Lamb-Oseen vortices, has to be used in combination with relations (6) and (25) for $s_{i} / s_{e}$ and $\cos \xi^{(n)}$. This formula is compared with experimental and numerical results in figure 13. The quantitative agreement is surprisingly good. It provides a strong validation of the theory on the elliptic instability mechanisms described above.

\subsection{Three-dimensional merging}

For moderate Reynolds numbers $(R e>2000)$, experiments show that as soon as the elliptic instability develops, the dynamics of the two vortices becomes strongly three-dimensional and departs from the two-dimensional dynamics described in section 2 . When the amplitude of the instability mode has reached sufficiently large values, a complex three-dimensional merging process of the vortices begins. It leads to the formation of a single vortex with specific characteristics. The three-dimensional merging process is much more complex than in two dimensions, as illustrated in figures 14(a-d). A strong disorder with small scales appears in the merging region, and three-dimensional secondary filament structures are ejected far from the vortices.

The three-dimensional merging process starts earlier than in two dimensions. This is in agreement with the mechanism discussed in section 2 : the instability deforms the vortices and can then move vorticity across the separatrix connected to the outer hyperbolic points (see figure 6). When this occurs, the vorticity is carried away outwards along the outer streamline; the two vortices then must get closer in order to conserve their angular momentum, and the merging process starts. In the experiments, three-dimensional merging has been found to occur for values of $(a / b)$ as small as 0.19 which has to be compared with the critical two-dimensional value of 0.22 .

After the merging, the final vortex reorganises into an axisymmetric structure. As for twodimensional flow, the vorticity profile of this structure is not Gaussian, but a core size can still be defined from the radius at which the azimuthal velocity reaches its maximum. Figure 15 shows the evolution of the square of the core size as a function of the viscous time for both cases, with and without instability. With instability, we see that not only the merging process starts earlier, but it also leads to a larger vortex. The core area is multiplied by a factor of 3.5 during the three-dimensional merging, while only a factor of 1.5 was observed in the two-dimensional case. 


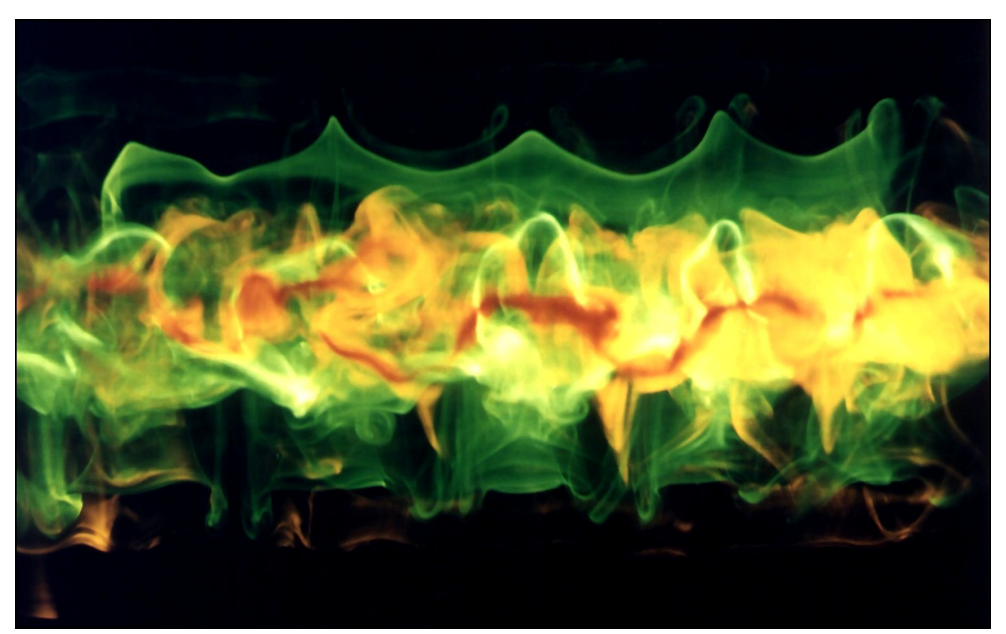

(a)

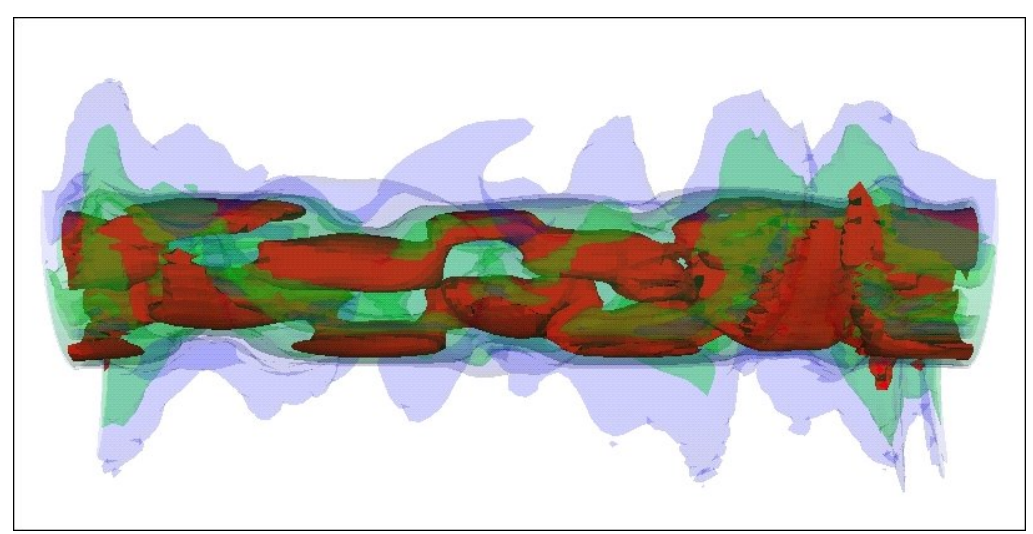

(c)

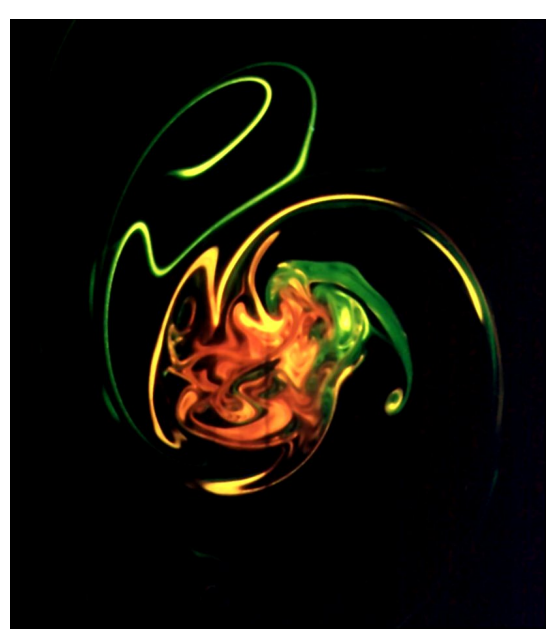

(b)

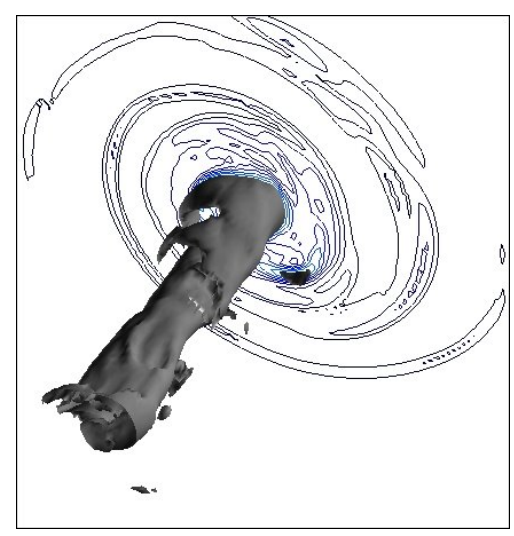

(d)

Fig. 14. Late stages of the elliptic instability after merging. Dye visualisations (a) in a sideview and (b) in a transverse section. (c-d) Vorticity isosurfaces from DNS, in perspective view and in a section. From [37].

The maximum velocity of the vortex is also smaller in the presence of the instability, it is less than $2 / 3$ of the value without instability. The velocity profiles are shown in figure 16. This is mainly due to a large decrease of the maximum vorticity by a factor of about 2.5, whereas it was conserved in the two-dimensional case. However, if we assume that the other conservation laws are still valid in three dimensions (circulation, energy and angular momentum), the profile of the final vortex can be predicted for the case shown in figure 16, using the vortex model composed of two concentric Gaussian vortices, as defined in (11), with the following parameters: $a_{c}=2.1985, \Gamma_{c}=1.9315, a_{f}=13.34$ and $\Gamma_{f}=0.0685$. This prediction is in good qualitative agreement with the experimental results, although a better agreement can be found using the two-core vortex defined by (16) with $\alpha=2 / 3, a_{1}=1.9$ and $a_{2}=6$. 


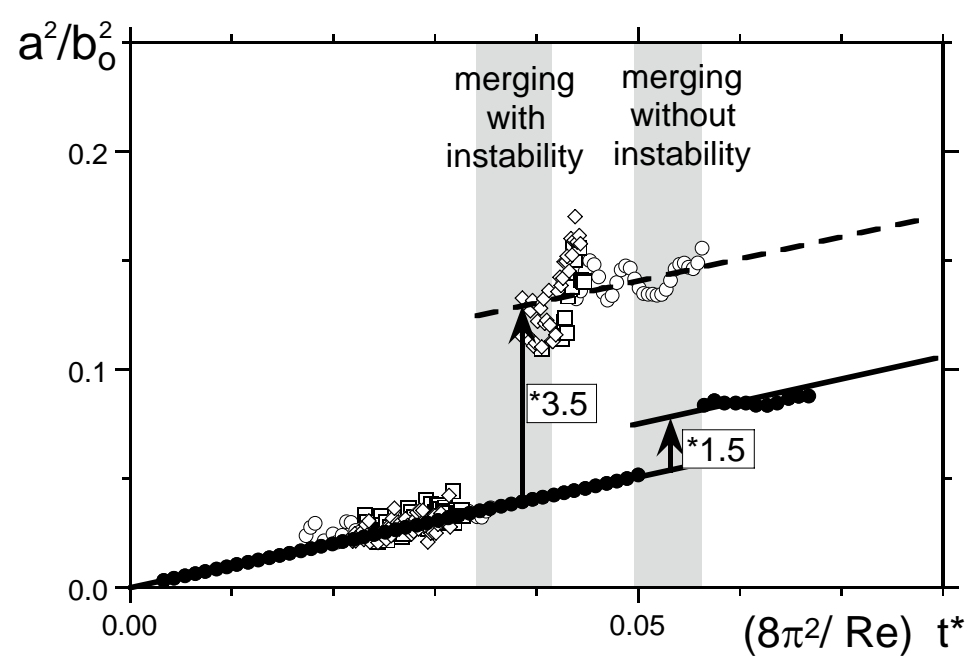

Fig. 15. Temporal evolution of the square of the non-dimensional core size. In the presence of the instability (open symbols), merging appears earlier and leads to a larger core than in the absence of the instability (filled symbols). Experiments: $R e=1506(\bullet) ; R e=3350$ (०); Re=5000 ( $\square, \diamond)$. Two-dimensional numerical results: $R e=8000$.
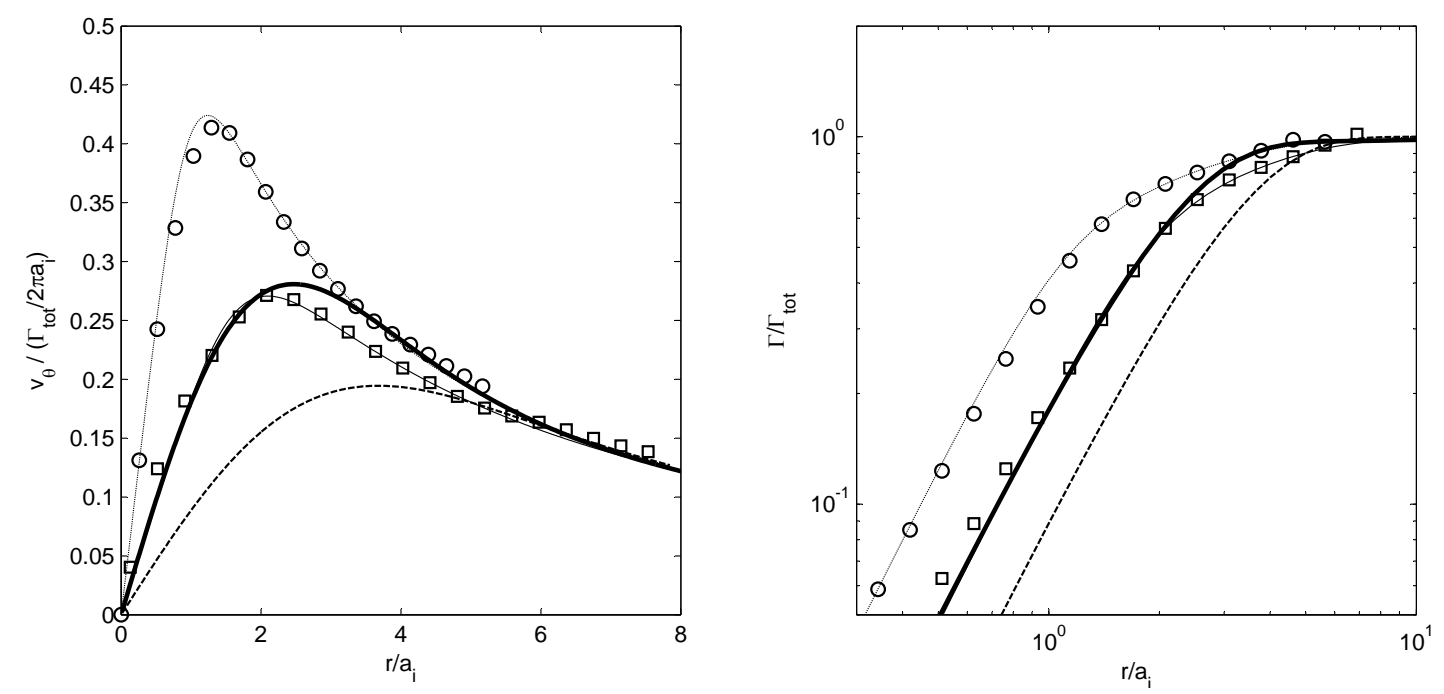

Fig. 16. Final azimuthal velocity (a) and local circulation (b) after the merging of the two vortices without instability (o) at $R e=1506$, and with instability $(\square)$ at $R e=3350$, obtained from experiments. Thin solid and dotted lines correspond to a fit using the two core model (16). The dashed line corresponds to a Gaussian vortex with conservation of circulation and angular momentum. The thick solid line corresponds to two concentric Gaussian vortices (11) with conservation of circulation, angular momentum and energy and a $60 \%$ loss of maximum vorticity. 
The three-dimensional merging initiated by the development of the elliptic instability has been obtained in numerical simulations up to $R e=5 \times 10^{4}$. For larger Reynolds numbers and smaller initial $a / b$, a different evolution has also been observed (F. Laporte, private communication). Under certain conditions, the elliptic instability has been seen to saturate and, after a transient disordered regime, leave the flow with two vortices, which are still distinct, but have much larger vortex cores. The elliptic instability was then observed to develop again, up to the beginning of the three-dimensional merging process as seen for smaller Reynolds numbers. No systematic study of this two-step three-dimensional merging has been performed, so it is difficult to infer the parameter range for which it is expected to occur. In particular, it is not excluded that, for even larger Reynolds numbers and smaller initial $a / b$, more than two steps of elliptic instability growth and saturation could be needed to reach the onset of merging.

The weakly nonlinear theory of the elliptic instability [44,45] provides some insight into the dynamics at large Reynolds numbers. For infinite Reynolds numbers, the theory predicts that the sinuous deformation associated with the two resonant Kelvin modes $m=1$ and $m=-1$ grows in the plane oriented along with the direction of stretching, then nonlinear effects make this plane rotate toward the direction of compression such that the deformation stops increasing, then decreases and goes back to zero [45]. As soon as viscous effects are considered, the weakly nonlinear dynamics is different. The most unstable deformation is expected to spiral to a fixed point of finite amplitude of order $\sqrt{s_{i} / \mu} \propto a / b$. Although such a spiraling evolution has been seen for the elliptic instability developing inside a cylinder [32], no clear evidence is available for the vortex pair. However, the saturation process has been observed, followed by a complex rearrangement in each vortex (probably due to secondary instabilities) by which new straight vortices are formed. If one assumes that, by this process, the core size of each vortex grows by a fraction of the amplitude deformation, i.e., a fraction of $a / b$, it is easy to imagine that, if $a / b$ is initially very small, several instability cycles would be needed to reach the critical ratio required for merging.

In conclusion, we have seen in this chapter that three-dimensional effects due to elliptic instability of the vortex cores strongly modify the merging process of co-rotating vortices. Three-dimensional merging sets in earlier (for smaller rescaled core sizes) than in two dimensions, it produces a more turbulent and larger final vortex, with greatly reduced maximum swirl velocity. The main characteristics of the final vortex can be predicted using a simple model involving the basic physical conservation laws. 


\section{Discussion}

In this paper, we have considered the temporal dynamics of two identical co-rotating Gaussian vortices without axial flow. This constitutes a very simple idealization of the near-wake dynamics of the vortices generated by a wing. In this section, we want to identify, as far as possible, the differences between the vortices in the near wake of an aircraft and our model, in order to determine the impact and relevance of the results presented in the previous sections for applications. This will allow us to discuss a few possible extensions of the theory to more realistic configurations, and to address some open issues.

The first difference to be pointed out is the fact that a flapped aircraft wing does not generate two "nice" identical Gaussian vortices. Even if two dominant vortices exist behind each wing, they are probably not identical, and they exhibit a large vortical region outside their viscous core, due to the roll-up of the vortex sheet. In addition, these two vortices may also be surrounded by other vortices, which are generated by various elements on the wing. These other vortices are expected to affect the two-dimensional dynamics of the vortex pair. Indeed, as soon as a third vortex is present, the two dominant vortices can get closer to each other due to the velocity field generated by this third vortex. The two vortices could therefore merge quickly even if their initial core size $(a / b)$ was very small. This scenario has not been considered above, where merging was always due to thickening of the vortex cores by either viscous diffusion or instability. It may be important in real configurations.

It is also worth mentioning that merging is not the only possibility of strong vortex interactions. When a small vortex gets close to a strong vortex, it is stretched and transformed into a vortex sheet which is wrapped around the larger vortex. This so-called straining phenomenon has been studied by Dritschel and Waugh [46,47] among others. It contributes in particular to the reorganization processes of the very near wake, during which the smallest vortices are wrapped around the largest ones.

The two-dimensional conditions for straining and merging are expected to be influenced by three-dimensional instabilities. In section 3.1, we have provided a theoretical model for the elliptic instability in two identical Gaussian vortices. When the two vortices possess different circulations or different core sizes, a similar model can be developed. Le Dizès and Laporte [29] showed that a formula for the instability growth rate can be obtained in each Gaussian vortex. When the vortices are not Gaussian, the elliptic instability is still expected to be active. Fabre and Jacquin [24] analyzed this possibility for the two-scale model defined in (16), by considering such a vortex in a weak stationary strain field. They demonstrated that both the growth rate and the selected wavelength depend on the profile parameters $a_{1} / a_{2}$ and $\alpha$. They showed the following interesting features: both the ratio $s_{i} / s_{e}$ of the strain rate in the vortex center, i.e., the maximum growth rate of the instability, and the width of the unstable wavenumber bands increase with the ratio $a_{2} / a_{1}$. They also identified two 
different regimes according to the value of $\alpha$. When $0.5<\alpha<1$, the unstable wavelengths were found to scale on the inner core size $a_{1}$, with values of $k_{z} a_{1}$ comparable to those for the Gaussian vortex. By contrast, for $\alpha<0.4$, the unstable wavelengths are much larger and scale on the outer core radius $a_{2}$. It was argued that, for these parameters, the elliptic instability is not governed by the local destabilization of the vortex center, but instead by the destabilization of the large intermediate region. Although only a stationary strain field was considered, it is reasonable to believe that similar conclusions would be reached in the case of a rotating strain field. In particular, for $\alpha>0.5$, we expect that a model similar to the one developed for the Gaussian vortex could be used for the prediction of the instability growth rate: expression (19) would still apply, but with modified relations for $s_{i} / s_{e}$ and $\cos \xi$. One can also conjecture that the presence of a large intermediate region could favor merging, since more vorticity can be advected outwards along the outer separatrix. Stronger vorticity arms would then be ejected far from the vortices, which could lead to a premature vortex merging, as explained in section 2.2. So far, however, there is no experimental or numerical evidence to confirm this expectation.

Wing-generated vortices are also characterized by an axial velocity component, due to a velocity deficit in the core region, with respect to the free-stream velocity transporting the vortices downstream. We now discuss some of the effects of this axial flow. First, it is important to note that axial flow does not modify the two-dimensional dynamics. Axial flow is passively advected by the two-dimensional velocity and diffused by viscosity. Therefore, the large scale two-dimensional dynamics, the vortex deformation, the viscous diffusion of the vortex cores and two-dimensional merging should not be influenced by axial flow. By contrast, axial flow is expected to strongly affect the three-dimensional dynamics. For strong axial flow, vortices can become unstable with respect to an helical instability [48]. For a Batchelor vortex (Gaussian profiles of axial velocity and vorticity), this happens when the axial flow parameter $W_{0}$, defined as the ratio of the maximum axial velocity difference and the maximum azimuthal velocity, exceeds approximatively 1 . It is not excluded that such large values of $W_{0}$ could be reached very close to the wing.

For small values of $W_{0}$, each vortex alone is expected to remain stable, so we can naturally address the question whether the elliptic instability is still active in those cases. This question is currently subject of active research. A few elements of response can be put forward. The first point to note is that the local theory of the elliptic instability should still apply in the vortex center, if we move with the axial velocity of the center. This means that expression (19) for the instability growth rate should be valid for the most unstable local perturbations located in the vortex center and moving with the flow. The problem is that we have now no guarantee that such a local perturbation can be compatible with the expression of a global instability mode. This can be seen by considering the effect of axial flow on the characteristics of the Kelvin waves. When there is an axial flow, the helical Kelvin waves $m=1$ and $m=-1$ are no longer symmetric. They do not possess the same 
frequency nor the same spatial structure. This implies that their combination does not form a stationary planar deformation any longer. Moreover, the symmetry breaking has the consequence that the characteristics of the resonant waves do not satisfy the local conditions (23), which permitted to use the local theory. The result is that the excitation of the Kelvin waves $m=1$ and $m=-1$ may become less efficient. This phenomenon has been analyzed in detail by Lacaze et al [49] for a Rankine vortex with axial flow. They demonstrated that other instability modes involving Kelvin waves with high azimuthal wavenumbers could become the most unstable, as $W_{0}$ increases. For a vortex with a continuous profile, such as the Batchelor vortex, there is another effect: some of the Kelvin waves which were involved in the resonance become damped and thus cannot be excited by the strain field. This situation has been studied by Lacaze [50] for a Batchelor vortex in a stationary strain field (see also [51]). He demonstrated that, because of the damping of the Kelvin wave $m=1$, the resonance between the two waves $m=1$ and $m=-1$ disappears as $W_{0}$ reaches a critical value. However, other pairs of waves which were not excited in the absence of axial flow are now excited by the strain field. In particular, Lacaze demonstrated that pairs of Kelvin waves $m=0$ and $m=-2$, and then $m=-1$ and $m=-3$ were successively excited, as $W_{0}$ increases. The instability mode composed of $m=0$ and $m=-2$ waves was apparently observed in numerical simulations of a counter-rotating Batchelor vortex pair by Laporte [52]. This observation has been recently confirmed (K. Ryan, private communication), and a quantitative comparison with the theoretical results is currently underway [53]. An analysis of these new instability modes in the case of co-rotating vortices is highly desirable, notably to determine their impact on the vortex merging phenomenon.

To close this section, we wish to address the effect of another important difference between real wake vortices and the vortices studied in this article. Wake vortices evolve spatially, whereas we have considered the temporal evolution of parallel vortices. We thus have to address the question whether our results can be applied locally in the frame moving with the mean stream $U$ as if the temporal dynamics were just advected at a constant speed. A priori, this is possible if the vortex system can be considered as locally parallel. This requires that the characteristic streamwise evolution length, which can be evaluated as $L=U t_{c} \approx 2 \pi^{2} b^{2} U / \Gamma$ if we take for $t_{c}$ the turnover time of the two vortices, is large compared to the largest characteristic transverse scale, given by the separation distance $b$. In practice, the ratio $L / b$ is larger than 30 for typical configurations, so it is reasonable to consider the spatial interaction of the two vortices as locally parallel. The two-dimensional merging occurs on a time scale comparable to $t_{c}$, so it should also be relevant for the spatial evolution.

Concerning the three-dimensional evolution, one also has to verify that the characteristic instability wavelength is small compared to $L$. This is of course satisfied for the elliptic instability as it scales on the core size $a$, which is smaller than $b$. As spatial evolution is considered, one should also analyze the spatio-temporal stability properties of the elliptic 


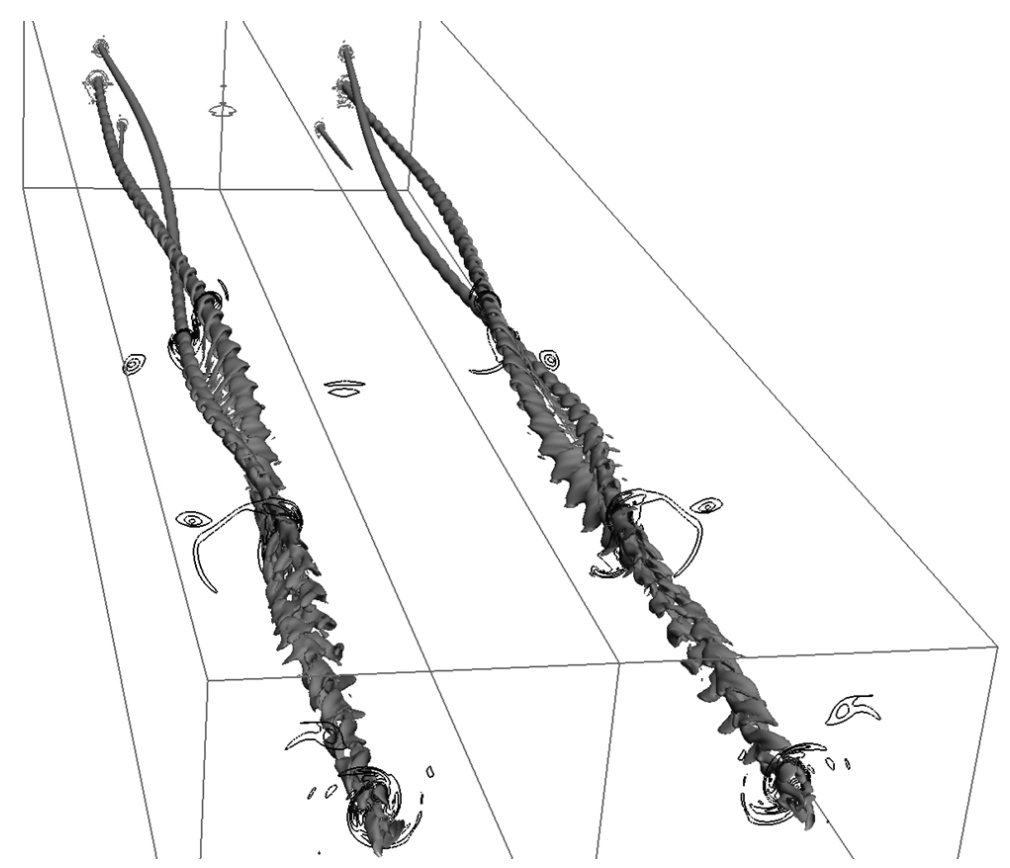

Fig. 17. Illustration of three-dimensional merging in a spatially evolving flow, representing a realistic aircraft wake. Vorticity contours from LES at $R e=10^{6}[52]$.

instability. This has been done for a model of counter-rotating vortices by Fabre et al [54]. They showed that the elliptic instability remains convective and that spatial growth rates can be deduced from temporal growth rates by a Gaster transformation, i.e., by assuming that the temporal mode is advected at the mean speed $U$. We expect similar conclusions for co-rotating vortices.

Finally, note that temporal results may not be relevant everywhere. In the very near wake, the dynamics is dominated by straining and vortex sheet roll-up which occur on a faster time scale than $t_{c}$. In this region, the locally parallel approximation is not expected to apply. Consequently, the very near wake characteristics are poorly described by a two-dimensional temporal approach.

Figure 17 shows the development of a short-wave perturbation on a spatially evolving vortex system with characteristic close to a real aircraft wake $\left(R e=10^{6}\right)$, a result obtained by Laporte [52] using Large-Eddy Simulation. It illustrates that elliptic instability of co-rotating vortices and the associated three-dimensional merging are indeed relevant for realistic applications.

One may mention that one aspect involved in the dynamics of real aircraft wakes has been completely excluded here, namely the influence of the atmosphere. The wake system of a flying aircraft, especially in the vicinity of airports, i.e., relatively close to the ground, is exposed to the influence of the surrounding atmosphere or atmospheric boundary layer. The 
effects of wind shear, stratification and atmospheric turbulence are particularly important (see, e.g., Holzäpfel et al. [55] and Mokry [56] for an overview).

\section{Conclusion}

In this paper, we have treated fundamental aspects related to the interaction of co-rotating vortices and the phenomenon of vortex merging. The basic two-dimensional analysis gave insight into the different phases of merging and their Reynolds number dependence. Simple models were presented, involving the basic conservation laws for circulation and energy, allowing to understand the physics of merging and to predict the properties of the final vortex resulting from this process. We have presented in detail a three-dimensional short-wave instability linked to the mutual elliptic deformation of the vortices, and strongly interfering with the two-dimensional merging. Its effects include a premature merging, a faster increase in core radius and a lower maximum swirl velocity for the final vortex, properties which are of potential interest in the context of wake vortex hazard reduction. Although the configurations considered in this paper may seem a somewhat severe simplification of realistic aircraft wakes during takeoff or landing, they nevertheless contain the most important features needed to understand the physics involved. The relevance of these results for applications were discussed, and it is anticipated that many of the conclusions drawn here will in some form carry over to the applied situation.

\section{Appendix}

We wish to calculate the energy of the two-dimensional flow before and after merging. Before merging, the flow is made of two Gaussian vortices with circulation $\Gamma$ and core size $a_{i}$, which are separated by a distance $b$. After merging, the flow is made of two concentric vortices with circulations $\Gamma_{c}$ and $\Gamma_{\text {fil }}$ and core sizes $a_{c}$ and $a_{\text {fil }}$. Since the total circulation is non zero, the kinetic energy of the flow is infinite and we need to use the excess kinetic energy, defined as:

$$
K_{e}=\lim _{R \rightarrow \infty} \int_{r<R} \frac{v^{2}}{2} \mathrm{~d} S-\frac{\Gamma_{\text {tot }}^{2}}{4 \pi} \ln \frac{R}{L}
$$

In this definition, $L$ is a characteristic length scale of the flow, and should be identical before and after merging. We will choose $L=a_{i}$ to simplify the calculations. Moreover, $\Gamma_{\text {tot }}$ is the total circulation of the flow and must be equal before and after merging. It is here equal to $2 \Gamma$ and to $\Gamma_{c}+\Gamma_{\text {fil }}$.

Introducing the velocity profile of two concentric Gaussian vortices into (27), it is easy to 
derive the energy of the flow after merging:

$$
K_{\mathrm{after}}=\Gamma_{c}^{2}\left[-C-\ln \left(\frac{a_{c}}{a_{i}}\right)\right]+\Gamma_{\mathrm{fil}}^{2}\left[-C-\ln \left(\frac{a_{\mathrm{fil}}}{a_{i}}\right)\right]+2 \Gamma_{c} \Gamma_{\mathrm{fil}}\left[K_{\mathrm{conc}}\left(\frac{a_{c}}{a_{\mathrm{fil}}}\right)-\ln \left(\frac{a_{c}}{a_{i}}\right)\right]
$$

where, $C$ is a constant defined as:

$$
C=\int_{0}^{1} \frac{\left(1-e^{-r^{2}}\right)^{2}}{4 \pi r} \mathrm{~d} r+\int_{1}^{\infty} \frac{e^{-2 r^{2}}-2 e^{-r^{2}}}{4 \pi r} \mathrm{~d} r \approx 4.6131 \times 10^{-4}
$$

and $K_{\text {conc }}\left(a_{c} / a_{\text {fil }}\right)$ is the mutual kinetic energy of two concentric vortices given by:

$K_{\text {conc }}\left(\frac{a_{1}}{a_{2}}\right)=\int_{0}^{1} \frac{\left(1-e^{-r^{2}}\right)\left(1-e^{-r^{2}\left(a_{1} / a_{2}\right)^{2}}\right)}{4 \pi r} \mathrm{~d} r+\int_{1}^{\infty} \frac{e^{-r^{2}} e^{-r^{2}\left(a_{1} / a_{2}\right)^{2}}-e^{-r^{2}}-e^{-r^{2}\left(a_{1} / a_{2}\right)^{2}}}{4 \pi r} \mathrm{~d} r$

To calculate the energy of the flow after merging, it is easier to use the definition of the kinetic excess energy using the vorticity and the streamfunction:

$$
K_{e}=\frac{1}{2} \int \Psi \omega \mathrm{d} S
$$

This definition is equivalent to Eq. (27) only if the constant of the streamfunction is chosen such that $\Psi$ is equivalent to $-\left(\Gamma_{\text {tot }} / 2 \pi\right) \ln (R / L)$ when $R$ tends to infinity. For two vortices separated by a distance $b$, the cross terms can be estimated by assuming that the streamfunction of one vortex is almost constant in the core of the opposite vortex, which leads to an approximation of the excess kinetic energy before merging:

$$
K_{\text {before }}=-2 \Gamma^{2} C-2 \Gamma^{2} K_{\text {sep }}\left(\frac{b}{a_{i}}\right)
$$

where the mutual kinetic energy of the two vortices is given by:

$$
K_{\mathrm{sep}}\left(\frac{b}{a}\right)=\int_{1}^{b / a} \frac{1-e^{-r^{2}}}{4 \pi r} \mathrm{~d} r+\int_{1}^{\infty} \frac{e^{-r^{2}}}{4 \pi r} \mathrm{~d} r
$$

This approximation is valid to six decimals for the critical merging ratio of $a / b=0.22$ and is accurate within $0.5 \%$ up to $a / b=0.4$. Equating (28) and (32) leads to the conservation of the energy given in (15).

\section{Acknowledgement}

Many of the results presented in this paper come from research conducted within the European project "C-Wake" (Wake Vortex Characterization and Control). The corresponding financial support of the European Union is gratefully acknowledged. 


\section{References}

[1] J. N. Reinaud, D. G. Dritschel, The critical merger distance between two co-rotating quasigeostrophic vortices, J. Fluid Mech. 522 (2005) 357-381.

[2] L. Jacquin, D. Fabre, D. Sipp, E. Coustols, Unsteadiness, instability and turbulence in trailing vortices, present volume (2005).

[3] D. W. Waugh, The efficiency of symmetric vortex merger, Phys. Fluids A 4 (8) (1992) 17451758.

[4] P. Meunier, U. Ehrenstein, T. Leweke, M. Rossi, A merging criterion for two-dimensional co-rotating vortices, Phys. Fluids 14 (8) (2002) 2757-2766.

[5] S. Le Dizès, A. Verga, Viscous interactions of two co-rotating vortices before merging, J. Fluid Mech. 467 (2002) 389-410.

[6] S. Le Dizès, Non-axisymmetric vortices in two-dimensional flows, J. Fluid Mech. 406 (2000) $175-198$.

[7] K. V. Roberts, J. P. Christiansen, Topics in computational fluid mechanics, Computer Phys. Comm. (suppl.) 3 (1972) 14-32.

[8] E. A. Overman, N. J. Zabusky, Evolution and merger of isolated vortex structures, Phys. Fluids 25 (8) (1982) 1297-1305.

[9] R. W. Griffiths, E. J. Hopfinger, Coalescing of geostrophic vortices, J. Fluid Mech. 178 (1987) 73-97.

[10] S. A. Brandt, J. D. Iversen, Merging of aircraft trailing vortices, J. of Aircraft 14 (12) (1977) $1212-1230$.

[11] V. J. Rossow, Convective merging of vortex cores in lift-generated wakes, J. of Aircraft 14 (3) (1977) 283-290.

[12] C. D. Winant, F. K. Browand, Vortex pairing : the mechanism of turbulent mixing layer growth at moderate Reynolds number, J. Fluid Mech. 63 (2) (1974) 237-255.

[13] K. Bajer, A. Bassom, A. Gilbert, Accelerated diffusion in the centre of a vortex, J. Fluid Mech. 437 (2001) 395-411.

[14] D. G. Dritschel, The stability and energetics of corotating uniform vortices, J. Fluid Mech. 157 (1985) 95-134.

[15] D. D. Dritschel, The nonlinear evolution of rotating configurations of uniform vortices, J. Fluid Mech. 172 (1986) 157-182.

[16] M. V. Melander, N. J. Zabusky, M. J. McWilliams, Symmetric vortex merger in two dimensions: Causes and conditions, J. Fluid Mech. 195 (1988) 303-340. 
[17] P. Saffman, R. Szeto, Equilibrium of a pair of equal uniform vortices, Phys. Fluids 23 (12) (1980) 2339-2342.

[18] C. Cerretelli, C. Williamson, The physical mechanism for vortex merging, J. Fluid Mech. 475 (2003) 41-77.

[19] P. Meunier, Etude expérimentale de deux tourbillons co-rotatifs, Ph.D. thesis, PhD Thesis, Université d'Aix-Marseille I, France (2001).

[20] P. B. Rhines, W. R. Young, How rapidly is a passive scalar mixed within closed streamlines, J. Fluid Mech. 133 (1983) 133-145.

[21] A. J. Bernoff, J. F. Lingevitch, Rapid relaxation of an axisymmetric vortex, Phys. Fluids 6 (11) (1994) 3717-3723.

[22] P. Meunier, T. Leweke, Elliptic instability of a co-rotating vortex pair, J. Fluid Mech. 533 $125-159,$.

[23] G. F. Carnevale, J. C. McWilliams, Y. Pomeau, J. B. Weiss, W. R. Young, Rates, pathways, and end states of nonlinear evolution in decaying two-dimensional turbulence: Scaling theory versus selective decay, Phys. Fluids A 4 (1992) 1314-1316.

[24] D. Fabre, L. Jacquin, Short-wave cooperative instabilities in representative aircraft vortices, Phys. Fluids 16 (5) (2004) 1366-1378.

[25] S. C. Crow, Stability theory for a pair of trailing vortices, AIAA J. 8 (12) (1970) 2172-2179.

[26] T. Leweke, C. H. K. Williamson, Cooperative elliptic instability of a vortex pair, J. Fluid Mech. 360 (1998) 85-119.

[27] J. Jiménez, Stability of a pair of co-rotating vortices, Phys. Fluids 18 (11) (1975) 1580-1581.

[28] P. Meunier, T. Leweke, Three-dimensional instability during vortex merging, Phys. Fluids 13 (10) (2001) 2747-2750.

[29] S. Le Dizès, F. Laporte, Theoretical predictions for the elliptic instability in a two-vortex flow, J. Fluid Mech. 471 (2002) 169-201.

[30] R. R. Kerswell, Elliptical instability, Ann. Rev. Fluid Mech. 34, 83-113.

[31] S. E. Widnall, D. Bliss, C.-Y. Tsai, The instability of short waves on a vortex ring, J. Fluid Mech. 66 (1) (1974) 35-47.

[32] C. Eloy, P. Le Gal, S. Le Dizès, Elliptic and triangular instabilities in rotating cylinders, J. Fluid Mech. 476 (2003) 357.

[33] B. J. Bayly, S. A. Orszag, T. Herbert, Instability mechanisms in shear-flow transition, Annu. Rev. Fluid Mech. 20 (1988) 359-391.

[34] T. Leweke, C. H. K. Williamson, Three-dimensional instabilities in wake transition, Eur. J Mech. B/Fluids 17 (1998) 571-586. 
[35] R. T. Pierrehumbert, Universal short-wave instability of two-dimensional eddies in an inviscid fluid, Phys. Rev. Lett. 57 (1986) 2157-2160.

[36] B. J. Bayly, Three-dimensional instability of elliptical flow, Phys. Rev. Lett. 57 (1986) 21602163.

[37] T. Leweke, P. Meunier, F. Laporte, D. Darracq, Controlled interaction of co-rotating vortices, in: K. Bütefisch et al. (Ed.), 3rd ONERA-DLR Aerospace Symposium (ODAS 2001), ONERA, Paris, 2001, paper S2-3.

[38] A. Lifschitz, E. Hameiri, Local stability conditions in fluid dynamics, Phys. Fluids A 3 (11) (1991) 2644-2651.

[39] S. Le Dizès, Three-dimensional instability of a multipolar vortex in a rotating flow, Phys. Fluids 12 (11) (2000) 2762-2774.

[40] D. W. Moore, P. G. Saffman, The instability of a straight vortex filament in a strain field, Proc. R. Soc. Lond. A. 346 (1975) 413-425.

[41] C.-Y. Tsai, S. E. Widnall, The stability of short waves on a straight vortex filament in a weak externally imposed strain field, J. Fluid Mech. 73 (4) (1976) 721-733.

[42] P. G. Saffman, Vortex dynamics, Cambridge University Press, 1992.

[43] D. Fabre, D. Sipp, L. Jacquin, The Kelvin waves of the Lamb-Oseen vortex, J. Fluid Mech. (submitted).

[44] F. Waleffe, The 3d instability of a strained vortex and its relation to turbulence, Ph.D. thesis, Massachusetts Institute of Technology (1989).

[45] D. Sipp, Weakly nonlinear saturation of short-wave instabilities in a strained Lamb-Oseen vortex, Phys. Fluids 12 (7) (2000) 1715-1729.

[46] D. G. Dritschel, D. W. Waugh, Quantification of the inelastic interaction of unequal vortices in two-dimensional vortex dynamics, Phys. Fluids A 4 (8) (1992) 1737-1744.

[47] R. R. Trieling, O. U. Velasco Fuentes, G. J. F. van Heijst, Interaction of two unequal nonuniform vortices, Phys. Fluids (submitted).

[48] R. L. Ash, M. R. Khorrami, Vortex stability, in: S. I. Green (Ed.), Fluid Vortices, Kluwer Academic Publishers, 1995, Ch. VIII, pp. 317-372.

[49] L. Lacaze, A.-L. Birbaud, S. Le Dizès, Elliptic instability in a Rankine vortex with axial flow, Phys. Fluids 17 (2005) 017101.

[50] L. Lacaze, L’instabilité elliptique: exemples en aéronautique et en géophysique, Ph.D. thesis, Université de Provence (2004).

[51] S. Le Dizès, L. Lacaze, An asymptotic description of vortex Kelvin modes, J. Fluid Mech. (in press). 
[52] F. Laporte, Simulation numérique appliquée à la caractérisation et aux instabilités des tourbillons de sillage des avions de transport, Ph.D. thesis, Institut National Polytechnique de Toulouse, CERFACS (2002).

[53] L. Lacaze, K. Ryan, S. Le Dizès, Elliptic instability in a batchelor vortex (in preparation).

[54] D. Fabre, C. Cossu, L. Jacquin, Spatio-temporal development of the long and short-wave vortex-pair instabilities, Phys. Fluids (2000) 1247-1250.

[55] F. Holzäpfel, T. Hofbauer, D. Darracq, H. Moet, F. Garnier, C. Ferreira Gago, Analysis of wake vortex decay mechanisms in the atmosphere, Aerosp. Sci. Technol. 7 (2003) 263-275.

[56] M. Mokry, The vortex merger factor in aircraft wake turbulence, Aeronaut. J. 109 (2005) $13-22$. 